\title{
NULL CONTROLLABILITY OF THE HEAT EQUATION IN UNBOUNDED DOMAINS BY A FINITE MEASURE CONTROL REGION*
}

\author{
Piermarco Cannarsa $^{1}$, Patrick Martinez ${ }^{2}$ and Judith Vancostenoble ${ }^{2}$
}

\begin{abstract}
Motivated by two recent works of Micu and Zuazua and Cabanillas, De Menezes and Zuazua, we study the null controllability of the heat equation in unbounded domains, typically $\mathbb{R}_{+}$ or $\mathbb{R}^{N}$. Considering an unbounded and disconnected control region of the form $\omega:=\cup_{n} \omega_{n}$, we prove two null controllability results: under some technical assumption on the control parts $\omega_{n}$, we prove that every initial datum in some weighted $L^{2}$ space can be controlled to zero by usual control functions, and every initial datum in $L^{2}(\Omega)$ can be controlled to zero using control functions in a weighted $L^{2}$ space. At last we give several examples in which the control region has a finite measure and our null controllability results apply.
\end{abstract}

Mathematics Subject Classification. 35K05, 93B05, 93B07.

Received May 20, 2002. Revised July 18, 2003.

\section{INTRODUCTION}

This paper is devoted to the study of the null controllability of the heat equation in an unbounded domain $\Omega$ by a "small" control region $\omega$.

We consider the problem

$$
\begin{cases}u_{t}-\Delta u=f \chi_{\omega}, & x \in \Omega, t \in(0, T), \\ u=0, & x \in \partial \Omega, t \in(0, T), \\ u(t=0, x)=u_{0}(x), & x \in \Omega .\end{cases}
$$

The control $f$ acts on the system through the open subset $\omega$, and $\chi_{\omega}$ denotes the characteristic function of $\omega$. The null controllability problem can be formulated as follows: given $T>0$ and $u_{0}$, does there exists a control $f$ such that the solution of (1.1) reaches the rest at time $T$, i.e. $u(T)=0$ ? On purpose, we do not precise for the moment the spaces where the initial condition $u_{0}$ and the control $f$ lie, since that will be one of the key points of this paper. We will also precise later what means a "small" control region.

Keywords and phrases. Null controllability, weighted observability inequalities.

* This work was done when the second and third authors were visiting the University Tor Vergata, Roma, invited by the University Tor Vergata, Roma, and the Istituto Nazionale di Alta Matematica. They wish to thank these institutions for their hospitality and financial support.

1 Dipartimento di Matematica, Università di Roma "Tor Vergata", Via della Ricerca Scientifica, 00133 Roma, Italy; e-mail: cannarsa@mat.uniroma2.it

2 Laboratoire M.I.P., UMR CNRS 5640, Université Paul Sabatier Toulouse III, 118 route de Narbonne, 31062 Toulouse Cedex

4, France; e-mail: martinez@mip.ups-tlse.fr; cancoste@mip.ups.tlse.fr 
First let us recall some of the main results on this question.

There is a large literature on this problem when $\Omega$ is a bounded set. Roughly speaking, if $\Omega$ is bounded and $\omega$ is any open subset of $\Omega$, then given $T>0$ and $u_{0} \in L^{2}(\Omega)$, there exists a control $f \in L^{2}\left(0, T ; L^{2}(\Omega)\right)$ such that the solution of (1.1) reaches the rest at time $T$. Similar results hold for boundary null controllability. See in particular Fattorini and Russell $[9,10]$ for similar results in one space dimension or under special geometric conditions, Lebeau and Robbiano [19] for a general result without geometric conditions, Fursikov and Imanuvilov [14] for similar results in a more general context, including heat equations with time-dependent coefficients, Fernández-Cara [11], Fernández-Cara and Zuazua [12,13] for similar results for semilinear heat equations.

When $\Omega$ is unbounded there are very few results, and the first one was negative: Micu and Zuazua [20] considered the case $\Omega:=\mathbb{R}_{+}^{*}=(0, \infty)$, and proved that, within the class of solutions defined by transposition, there is no smooth compactly supported initial datum that might be driven to zero in finite time by a control acting at the extremity $x=0$. Similar results hold when $\Omega$ is the half space $\mathbb{R}_{+}^{n}$ and when the control is distributed in a bounded control region $\omega$ (see Micu and Zuazua [21]). The lack of null controllability comes from the fact that the controlled heat equation holds in an unbounded domain, while the control is localized on a bounded domain, thus an unbounded region is left without control.

Very recently, Cabanillas, De Menezes and Zuazua [3] studied the problem of null controllability when the domain $\Omega$ is unbounded but when the control acts on a large control region $\omega$ : under the assumption

$$
\Omega \backslash \omega \text { is bounded, }
$$

they obtained positive results: given $T>0$ and $u_{0} \in L^{2}(\Omega)$, there exists a control $f \in L^{2}\left(0, T ; L^{2}(\Omega)\right)$ such that the solution of (1.1) reaches the rest at time $T$. (More generally, they obtained similar results for the semilinear heat equation, with nonlinearities involving gradient terms.)

We are interested in the null controllability properties when $\Omega$ is unbounded. Comparing all these results, it seems natural to ask if it possible to control the heat equation with a control acting on an unbounded region of finite measure, which would be the "smallest" control region that one can hope to be efficient to obtain null controllability results. This is the goal of this paper.

We are mainly interested in the case of $\Omega:=(0,+\infty)$. Thus we consider

$$
\begin{cases}u_{t}-u_{x x}=f \chi_{\omega}, & x \in(0, \infty), t \in(0, T), \\ u(t, x=0)=0, & t \in(0, T), \\ u(t=0, x)=u_{0}(x), & x \in(0,+\infty) .\end{cases}
$$

The control region is $\omega:=\cup_{n}\left(a_{n}, b_{n}\right)$, where the intervals $\omega_{n}:=\left(a_{n}, b_{n}\right)$ are disjoint and $a_{n} \rightarrow \infty$ as $n \rightarrow \infty$. Our main results are the following: under some assumption on the lengths of the control sets $\omega_{n}$ and the lengths of the uncontrolled sets $\left(b_{n}, a_{n+1}\right)$, we obtain two controllability results:

- first, if the initial datum $u_{0}$ lies in some weighted space $L_{\rho_{1}}^{2}(\Omega)$ (for some weight function $\rho_{1}$ that depends on the sequences $\left(a_{n}\right)_{n}$ and $\left.\left(b_{n}\right)_{n}\right)$, then there exists a control $f \in L^{2}\left(0, T ; L^{2}(\Omega)\right)$ such that $u(T)=0$. In particular, if $u_{0}$ is compactly supported, then $u_{0} \in L_{\rho_{1}}^{2}(\Omega)$ and our result applies. Hence this is a null controllability result by usual control functions for a class of initial data that is smaller than in the usual case;

- next, we prove that for all $u_{0} \in L^{2}(\Omega)$, there exists a control in a larger class: $f \in L^{2}\left(0, T ; L_{\rho_{2}}^{2}(\Omega)\right)($ for some weight function $\rho_{2}$ that depends on the sequences $\left(a_{n}\right)_{n}$ and $\left.\left(b_{n}\right)_{n}\right)$ such that $u(T)=0$ (in the space $L_{\rho_{2}}^{2}(\Omega)$ ). Hence this is a null controllability result for all the usual initial data but by controls in a larger class than the usual $L^{2}$ space.

The proofs of these results are based on suitable weighted minimization problems. The corresponding weighted observability inequalities are obtained cutting off the solutions in several pieces: roughly speaking, the ones in the control region and the ones in the uncontrolled parts $\left(b_{n}, a_{n+1}\right)$, which are estimated via the usual Carleman 
estimates on bounded sets. The technical assumption that we need is on the growth at infinity of the constants that appear in the Carleman estimates, that depend on the sequences $\left(a_{n}\right)_{n}$ and $\left(b_{n}\right)_{n}$, and means that the uncontrolled parts are "not too large" with respect to the controlled ones.

At last, we discuss some examples that satisfy our technical assumption on the sequences $\left(a_{n}\right)_{n}$ and $\left(b_{n}\right)_{n}$. For instance, our theory can be applied if the lengths of the uncontrolled parts are bounded above and below, and if the lengths of the controlled parts $\omega_{n}=\left(a_{n}, b_{n}\right)$ go to zero at most exponentially, that is

$$
0<m \leq a_{n+1}-b_{n} \leq M<\infty \quad \text { and } \quad\left|\omega_{n}\right| \geq c \mathrm{e}^{-c^{\prime} n} \quad \forall n \in \mathbb{N}
$$

for some positive constants $c, c^{\prime}$. Another interesting situation is when the lengths of the uncontrolled parts go to infinity at infinity in such a way that

$$
m n \leq a_{n+1}-b_{n} \leq M n \quad \forall n \in \mathbb{N} .
$$

Then, estimating the constants that appear in the Carleman estimates when the diameter of the domain goes to infinity, we prove that the technical assumption we need is satisfied under the very weak condition

$$
\forall n \in \mathbb{N},\left|\omega_{n}\right| \geq c \mathrm{e}^{-c^{\prime} n^{2}} .
$$

The outline of this paper is the following. In Section 2 we describe our main results, as well as open questions and possible extensions. In Section 3 we provide proofs of all our theoretical results, while applications and examples are discussed in Section 4. In Section 5, we show how to extend our results to a $N$ dimensional problem.

\section{MAin RESUlts}

\subsection{Null controllability results in one space dimension}

We set $\Omega:=(0,+\infty), \omega_{n}:=\left(a_{n}, b_{n}\right)$ and $\omega:=\cup_{n} \omega_{n}$ ( $\omega$ is the control region). We assume that the intervals $\left(a_{n}, b_{n}\right)$ are disjoint $\left(\right.$ i.e. $\left.0=b_{0}<a_{1}<b_{1}<a_{2}<b_{2}<\cdots\right)$ and $a_{n} \rightarrow \infty$ as $n \rightarrow \infty$.

Our first result concerns the problem:

$$
\begin{cases}w_{t}+w_{x x}=0, & x \in \Omega, t \in(0, T) \\ w(t, x=0)=0, & t \in(0, T) \\ w(T) \in L^{2}(\Omega) & \end{cases}
$$

(As we will see later, (2.1) is not always the adjoint equation of (1.2) because of the presence of a weight function in the norm.) We prove the following observability inequalities, that will be the main tools for our null controllability results:

Proposition 2.1. Given $T>0$, there exist two bounded functions $\rho_{1}, \rho_{2}: \mathbb{R}_{+} \rightarrow \mathbb{R}_{+}^{*}$ of class $\mathcal{C}^{1}$, depending on $\Omega, \omega$ and $T$, such that the solution $w(t, x)$ of (2.1) satisfies:

$$
\int_{T / 4}^{3 T / 4} \int_{\Omega} w(t, x)^{2} \rho_{1}(x) \mathrm{d} x \mathrm{~d} t \leq \int_{0}^{T} \int_{\omega} w(t, x)^{2} \mathrm{~d} x \mathrm{~d} t,
$$

and

$$
\int_{T / 4}^{3 T / 4} \int_{\Omega} w(t, x)^{2} \mathrm{~d} x \mathrm{~d} t \leq \int_{0}^{T} \int_{\omega} w(t, x)^{2} \frac{\mathrm{d} x}{\rho_{2}(x)} \mathrm{d} t
$$


Remarks 1. Micu and Zuazua [20] (p. 1637) noted that this kind of estimate is false when the control region $\omega$ is bounded: there is no positive weight function $\rho$ such that

$$
\int_{\Omega} w(0, x)^{2} \rho(x) \mathrm{d} x \leq C \int_{0}^{T} w_{x}(t, 0)^{2} \mathrm{~d} t
$$

for every smooth solution of (2.1). Similarly there is no positive weight function $\rho$ such that

$$
\int_{\Omega} w(0, x)^{2} \rho(x) \mathrm{d} x \leq C \int_{0}^{T} \int_{\omega} w(t, x)^{2} \mathrm{~d} x \mathrm{~d} t
$$

for every smooth solution of (2.1) when $\omega$ is bounded. However Cabanillas, De Menezes and Zuazua [3] proved that (2.4) is true with $\rho \equiv 1$ when $\Omega \backslash \omega$ is bounded. Hence our estimates are intermediate between the negative result when $\omega$ is bounded and the positive result when $\Omega \backslash \omega$ is bounded.

2. The construction of $\rho_{1}$ and $\rho_{2}$ allows us to estimate their behavior as $x \rightarrow \infty$. In particular we will see that if the length of the controlled parts $\omega_{n}$ is bounded below: $\left|\omega_{n}\right|=b_{n}-a_{n} \geq m>0$, and if the length of the uncontrolled parts is bounded above: $a_{n+1}-b_{n} \leq M$, then the functions $\rho_{1}$ and $\rho_{2}$ can be chosen constant. The problem becomes more interesting when $\left|\omega_{n}\right|=b_{n}-a_{n} \rightarrow 0$ as $n \rightarrow \infty$ and $m \leq a_{n+1}-b_{n} \leq M$. In that case $\rho_{1}(x) \rightarrow 0$ and $\rho_{2}(x) \rightarrow 0$ as $x \rightarrow \infty$. In this case, it is not clear that $w / \sqrt{\rho_{2}} \in L^{2}(\Omega \times(0, T))$. This will be clarified later, as well as the asymptotic behavior of $\rho_{1}$ and $\rho_{2}$ at infinity.

To deduce controllability results from Proposition 2.1 we need the following

Main assumption: the functions $\rho_{1}$ and $\rho_{2}$ satisfy the following differential inequality: there exists positive constants $\alpha_{1}$ and $\alpha_{2}$ such that

$$
\forall x,\left|\rho_{1, x}\right| \leq \alpha_{1} \rho_{1}, \quad \text { and } \quad\left|\rho_{2, x}\right| \leq \alpha_{2} \rho_{2}
$$

Roughly speaking, this means that the functions $\rho_{1}$ and $\rho_{2}$ do not decay too fast to zero at infinity (not faster than some $\left.\mathrm{e}^{-c x}\right)$. Since the behavior at infinity of $\rho_{1}$ and $\rho_{2}$ is closely related to the sequences $a_{n}$ and $b_{n}$, this means that the uncontrolled parts are "not too large" with respect to the controlled parts. This kind of technical assumption is quite necessary: indeed, it seems reasonable to think that the faster the lengths of the controlled parts go to zero, the stronger the control has to be on every $\omega_{n}$ to be efficient, and hence it belongs to some weighted space. On the other hand, some restrictions appear on the growth of $f$ to solve in a correct sense the heat equation (see Jones [16]).

Let us introduce the space

$$
L_{\rho_{2}}^{2}(\Omega):=\left\{v: \Omega \rightarrow \mathbb{R}, \int_{\Omega}|v|^{2} \rho_{2}(x) \mathrm{d} x<\infty\right\} .
$$

Of course, endowed with the scalar product

$$
\langle u, v\rangle_{\rho_{2}}:=\int_{\Omega} u v \rho_{2}(x) \mathrm{d} x
$$

$L_{\rho_{2}}^{2}(\Omega)$ is an Hilbert space. We introduce also the space

$$
H_{\rho_{2}}^{2}(\Omega):=\left\{v \in L_{\rho_{2}}^{2}(\Omega) \text { s.t. } u_{x}, u_{x x} \in L_{\rho_{2}}^{2}(\Omega)\right\}
$$

Denote $\tilde{A}$ the unbounded operator $\frac{\mathrm{d}^{2}}{\mathrm{~d} x^{2}}$ with the Dirichlet boundary condition $u(x=0)=0$. The domain of $\tilde{A}$ is

$$
D(\tilde{A}):=\left\{u \in H_{\rho_{2}}^{2}(\Omega) \text { s.t. } u(x=0)=0\right\} .
$$


Standard arguments can be used to justify that, if $\rho_{2}$ satisfies the differential inequality (2.5), then $\tilde{A}$ is the generator of an analytic semi-group in $L_{\rho_{2}}^{2}(\Omega)$. Therefore, given $u_{0} \in L_{\rho_{2}}^{2}(\Omega)$ and $f \in L^{2}\left(0, T ; L_{\rho_{2}}^{2}(\Omega)\right.$ ), problem (1.2) has a unique mild solution $u^{f} \in C\left([0, T] ; L_{\rho_{2}}^{2}(\Omega)\right)$.

Now we are ready to state our main null controllability results:

Theorem 2.1. Fix $T>0$. Under assumption (2.5), the following holds:

(i) if $u_{0} \rho_{1}^{-1 / 2} \in L^{2}(\Omega)$ (or equivalently if $u_{0} \in L_{1 / \rho_{1}}^{2}(\Omega)$ ), then there exists a control $f \in L^{2}\left(0, T ; L^{2}(\Omega)\right.$ ) such that the solution $u$ of $(1.2)$ satisfies $u^{f}(T)=0$;

(ii) if $u_{0} \in L^{2}(\Omega)$, there exists a control $f \in L^{2}\left(0, T ; L_{\rho_{2}}^{2}(\Omega)\right)$ such that the solution $u$ of (1.2) satisfies $u^{f}(T)=0$ (in the space $\left.L_{\rho_{2}}^{2}(\Omega)\right)$.

For most of the computations that follow we will need to consider more regular solutions, that is $u^{f} \in$ $L^{2}(0, T ; D(\tilde{A})) \cap H^{1}\left(0, T ; L_{\rho_{2}}^{2}(\Omega)\right)$. Such a regularity is guaranteed if the initial conditions are smoother, for instance if

$$
u_{0} \in H_{\rho_{2}}^{1}(\Omega):=\left\{u \in L_{\rho_{2}}^{2}(\Omega): u_{x} \in L_{\rho_{2}}^{2}(\Omega), u(x=0)=0\right\} .
$$

On the other hand, this fact will imply no loss of generality since we are mainly interested in $L^{2}$-estimates for the solution that can be easily recovered by an approximation argument.

Now it is interesting to exhibit examples where the technical assumption (2.5) is satisfied.

Example 1. As noted in Remark 2, if the lengths of the uncontrolled parts are bounded above:

$$
a_{n+1}-b_{n} \leq M<\infty,
$$

and if the lengths of the controlled parts $\omega_{n}=\left(a_{n}, b_{n}\right)$ are bounded below:

$$
\forall n, b_{n}-a_{n} \geq m>0,
$$

then the functions $\rho_{1}$ and $\rho_{2}$ can be chosen constant. Hence Theorem 2.1 applies, and gives "usual" null controllability results: given $u_{0} \in L^{2}(\Omega)$, there exists $f \in L^{2}\left(0, T ; L^{2}(\Omega)\right)$ such that the solution $u$ of $(1.2)$ satisfies $u(T)=0$. This refines the result of [3] in the linear case. (However note that the total measure of the control region $\omega$ is still infinite in this case.)

Now we are interested in finding finite measure control sets. Hence in Examples 2 and 3, we assume that the lengths of the uncontrolled parts are bounded below. In Example 2 we assume that they are also bounded above, in Example 3 we assume that they go to infinity at infinity.

Example 2. Property (2.5) holds true for instance if the lengths of the uncontrolled parts are bounded below and above and if the lengths of the controlled parts $\omega_{n}=\left(a_{n}, b_{n}\right)$ go to zero at most exponentially, that is

$$
\begin{gathered}
0<m \leq a_{n+1}-b_{n} \leq M<\infty, \\
b_{n}-a_{n} \geq c \mathrm{e}^{-c^{\prime} n},
\end{gathered}
$$

for every $n$ and for some positive constants $m, M, c, c^{\prime}$. Hence this provides a class of situations for which the control region has a finite measure (for example if $b_{n}-a_{n}=\mathrm{e}^{-n}$ ) and the two null controllability results hold.

Example 3. The other interesting situation is when the lengths of the uncontrolled parts go to infinity at infinity: estimating the constants that appear in the Carleman estimates, we prove that if

$$
m n^{\alpha} \leq a_{n+1}-b_{n} \leq M n^{\beta}
$$


with $0 \leq \alpha \leq \beta \leq 1$, and $2 \beta \leq 1+\alpha$, then the technical assumption that we need is satisfied if

$$
\forall n,\left|\omega_{n}\right| \geq c \mathrm{e}^{-c^{\prime} n^{1+\alpha}} .
$$

For example if $\alpha=1=\beta$, then under the very weak condition

$$
\forall n,\left|\omega_{n}\right| \geq c \mathrm{e}^{-c^{\prime} n^{2}}
$$

the two null controllability hold (and the control set $\omega$ may have a finite measure).

At first glance, the above discussion may seem a little paradoxal: in Example 3, the uncontrolled parts are larger than in Example 2, nevertheless the (sufficient) technical assumption on the controlled parts is weaker. Such an observation, however, takes into account just the existence of controls that steer our system to zero, without paying any attention to other possible properties, such as the norm of such controls. This may raise the question of finding the "best control location", namely the choice of the family of intervals $\left\{\omega_{n}\right\}$ that optimizes some given criterion.

These three examples satisfy the following general conditions:

$$
\left\{\begin{array}{l}
\left(a_{n+1}-b_{n}\right)^{2} \leq r a_{n} \\
\left|\omega_{n}\right| \geq r^{-1} \mathrm{e}^{-r a_{n}}
\end{array}\right.
$$

for some constant $r$. We prove that under these conditions, our assumption (2.5) is satisfied, and thus our null controllability results hold. Note that the first condition gives a bound on the length of the uncontrolled parts (they must be "not too large"), the second gives a bound on the length of the control parts (they must be "not too small"), in both cases the bound is given by the position of the control part $\left(a_{n}, b_{n}\right)$.

\subsection{Null controllability results in $N$ space dimension}

Our results are not restricted to one space dimension. For example consider $\Omega:=\mathbb{R}^{N}$, consider (as previously) two real sequences $\left(a_{n}\right)_{n}$ and $\left(b_{n}\right)_{n}$ such that $0=b_{0}<a_{1}<b_{1}<a_{2}<\cdots$ and $a_{n} \rightarrow \infty$ as $n \rightarrow \infty$, and define the controlled parts $\omega_{n}:=\left\{x \in \Omega,|x| \in\left(a_{n}, b_{n}\right)\right\}$, the control set $\omega:=\cup_{n} \omega_{n}$, and the uncontrolled parts $\mathcal{O}_{n}:=\left\{x \in \Omega,|x| \in\left(b_{n}, a_{n+1}\right)\right\}$. Then the uncontrolled set $\Omega \backslash \omega$ is composed by bounded connected components. Of course this is a $N$ dimensional situation that looks like the one dimensional problem we studied before. In this case, all our results remain valid. More precisely, consider $w$ the solution of

$$
\left\{\begin{array}{l}
w_{t}+\Delta w=0, x \in \Omega, t \in(0, T) \\
w(T) \in L^{2}(\Omega) .
\end{array}\right.
$$

Then the following holds:

Proposition 2.2. Given $T>0$, there exist two bounded functions $\rho_{1}, \rho_{2}: \Omega \rightarrow \mathbb{R}_{+}^{*}$ of class $\mathcal{C}^{1}$, depending on the sequences $\left(a_{n}\right)_{n}$ and $\left(b_{n}\right)_{n}$ and on $T$, such that the solution $w(t, x)$ of $(2.7)$ satisfies:

$$
\int_{T / 4}^{3 T / 4} \int_{\Omega} w(t, x)^{2} \rho_{1}(x) \mathrm{d} x \mathrm{~d} t \leq \int_{0}^{T} \int_{\omega} w(t, x)^{2} \mathrm{~d} x \mathrm{~d} t,
$$

and

$$
\int_{T / 4}^{3 T / 4} \int_{\Omega} w(t, x)^{2} \mathrm{~d} x \mathrm{~d} t \leq \int_{0}^{T} \int_{\omega} w(t, x)^{2} \frac{\mathrm{d} x}{\rho_{2}(x)} \mathrm{d} t .
$$

(Of course under our geometrical assumptions, the weight functions $\rho_{1}$ and $\rho_{2}$ are radial.)

Then in the same way, one easily verifies the following generalization of Theorem 2.1: 
Theorem 2.2. Fix $T>0$. Consider the control problem

$$
\begin{cases}u_{t}-\Delta u=f \chi_{\omega}, & x \in \Omega, t \in(0, T) \\ u(t=0, x)=u_{0}(x), & x \in \Omega\end{cases}
$$

Then

(i) assume that $\left|\nabla \rho_{1}\right| \leq \alpha_{1} \rho_{1}$ for some $\alpha_{1}$; then if $u_{0} \rho_{1}^{-1 / 2} \in L^{2}(\Omega)$ (or equivalently if $u_{0} \in L_{1 / \rho_{1}}^{2}(\Omega)$ ), then there exists a control $f \in L^{2}\left(0, T ; L^{2}(\Omega)\right)$ such that the solution $u$ of $(2.10)$ satisfies $u^{f}(T)=0$;

(ii) assume that $\left|\nabla \rho_{2}\right| \leq \alpha_{2} \rho_{2}$ for some $\alpha_{2}$; then if $u_{0} \in L^{2}(\Omega)$, there exists a control $f \in L^{2}\left(0, T ; L_{\rho_{2}}^{2}(\Omega)\right)$ such that the solution $u$ of $(2.10)$ satisfies $u^{f}(T)=0$ (in the space $L_{\rho_{2}}^{2}(\Omega)$ ).

Note that Theorem 2.2 directly follows from the observability inequalities stated in Proposition 2.2. These inequalities are obtained thanks to the essential property that the uncontrolled parts $\mathcal{O}_{n}$ are bounded, using suitable cut-off functions and Carleman estimates. Then it remains to study the growth of the weight functions with respect to the sequences $\left(a_{n}\right)_{n}$ and $\left(b_{n}\right)_{n}$. This is done estimating the constants that appear in the Carleman estimates, especially looking to the influence of the size of the control set and the size of the domain on the constants. It is in this part that such simple geometric conditions (we consider the radial situation) are interesting. However our results remain valid if we assume that the controlled parts $\omega_{n}$ contain the part $\left\{x \in \Omega,|x| \in\left(a_{n}, b_{n}\right)\right\}$ (in this case, we only use these subparts to control the equation). Carleman estimates are a little more complicated to establish in the $N$ dimensional case, however we prove that the constants that appear are the same than in the one dimensional case. Hence the functions $\rho_{1}$ and $\rho_{2}$ are the same than in the one dimensional case. Since they are radial, the needed growth assumptions $\left|\nabla \rho_{1}\right| \leq \alpha_{1} \rho_{1}$ and $\left|\nabla \rho_{2}\right| \leq \alpha_{2} \rho_{2}$ are satisfied under the assumptions corresponding to Examples 1-3:

$$
\left\{\begin{array} { l } 
{ b _ { n } - a _ { n } \geq m , } \\
{ a _ { n + 1 } - b _ { n } \leq M , }
\end{array} \quad \left\{\begin{array} { l } 
{ b _ { n } - a _ { n } \geq c \mathrm { e } ^ { - c ^ { \prime } n } , } \\
{ m \leq a _ { n + 1 } - b _ { n } \leq M , }
\end{array} \quad \left\{\begin{array}{l}
b_{n}-a_{n} \geq c \mathrm{e}^{-c^{\prime} n^{1+\alpha}} \\
m n^{\alpha} \leq a_{n+1}-b_{n} \leq M n^{\beta},
\end{array}\right.\right.\right.
$$

(with the same restriction on $\alpha$ and $\beta$ ). Hence one again this provides a whole range of admissible control sets of finite measure.

\subsection{Extensions and open questions}

Semilinear problems. In [3], the authors consider a semilinear heat equation. One expects that their fixed point method could be applied in the situation that we consider, but this would have to be checked.

Null controllability results in $N$ space dimension. The main problem is the following: consider a sequence $\left(\Omega_{n}\right)_{n}$ of smooth bounded domains in $\mathbb{R}^{N}$, and a sequence of open subsets $\left(\omega_{n}\right)_{n}, \omega_{n} \subset \Omega_{n}$. Now, given $T>0$, consider the solution of the heat equation:

$$
\begin{cases}u_{t}^{n}-\Delta u^{n}=0, & x \in \Omega_{n}, t \in(0, T) \\ u^{n}=0, & x \in \partial \Omega_{n} \\ u^{n}(0, x)=u_{0}^{n}(x) & \end{cases}
$$

Then we know [14] that there exists some $C\left(\Omega_{n}, \omega_{n}, T\right)>0$ such that

$$
\int_{T / 4}^{3 T / 4} \int_{\Omega_{n}} u(t, x)^{2} \mathrm{~d} t \mathrm{~d} x \leq C\left(\Omega_{n}, \omega_{n}, T\right) \int_{0}^{T} \int_{\omega_{n}} u(t, x)^{2} \mathrm{~d} x \mathrm{~d} t .
$$

The question that has to be studied is: what is the behavior of the constant $C\left(\Omega_{n}, \omega_{n}, T\right)$ when the size of $\Omega_{n}$ goes to infinity, or the size of the control region $\omega_{n}$ goes to zero (or both cases)? This behavior is closely related to 
the behavior at infinity of our weight functions. Here we study the one-dimensional case when $\Omega_{n}=\left(c_{n}, c_{n+1}^{\prime}\right)$, $\omega_{n}=\left(c_{n}, d_{n}\right) \cup\left(d_{n+1}^{\prime}, c_{n+1}^{\prime}\right)$ with $c_{n}<d_{n}<d_{n+1}^{\prime}<c_{n+1}^{\prime}$, and

- $\left|\Omega_{n}\right|$ remains bounded below and above and $\left|\omega_{n}\right| \rightarrow 0$ as $n \rightarrow \infty$;

$-\left|\Omega_{n}\right| \rightarrow \infty$ and $\left|\omega_{n}\right| \rightarrow 0$ as $n \rightarrow \infty$.

We extend also this study to the radial case in $N$ space dimension. We provide estimates of the constant $C\left(\Omega_{n}, \omega_{n}, T\right)$ (see Lems. 3.1-3.3) using Carleman estimates. Improving these estimates in the one-dimensional case would lead to an improvement of our results. And it would be interesting to study more general geometric situations in the $N$ dimensional case. Note that Fernandez-Cara and Zuazua [12,13] specially studied the dependence of the observability constants with respect to time and an additional potential term.

Regional null controllability. We give in this paper a result that can be seen as the "best" positive result of (global) null controllability that can be expected between the negative result of Micu and Zuazua [20] and the positive result of Cabanillas, De Menezes and Zuazua [3]. Indeed we provide a result of (global) null controllability with an unbounded control region of finite measure. This is the "smallest" control region that allows to obtain a positive result of (global) null controllability. In particular, our result is a refinement of the result of [3].

On the other hand, introducing the notion of regional null controllability, we improved the result of [3] in another direction (see [4]): when the control acts on a bounded region $\omega:=(\alpha, \beta)$ with $0<\alpha<\beta \leq \infty$, then Micu and Zuazua [20] proved that there is no smooth compactly supported initial datum that might be driven to zero in finite time. In [4], we proved that it is possible to drive the solution $u$ of (1.2) to zero on some subinterval. More precisely, given $\delta>0$, there exists $f \in L^{2}(\omega \times(0, T))$ such that the solution $u$ of $(1.2)$ satisfies $u(T)=0$ on the interval $(0, \beta-\delta)$. In particular, if $\beta=+\infty$ (which is the typical example for which the condition of [3] holds: $\Omega \backslash \omega$ is bounded), then $u(T)=0$ on the whole domain $(0, \infty)$. But if $\beta<\infty$, this gives a result of null controllability in some subdomain of $\Omega$, in a case for which it is not possible to obtain null controllability on the whole domain.

\section{Proof of Theorem 2.1}

The proof of Theorem 2.1 follows from Proposition 2.1, considering for each null controllability result an adequate minimization problem.

\subsection{Proof of Proposition 2.1}

We first prove the following

Lemma 3.1. For all $n$, there exists $C_{n}$ and $\tilde{C}_{n+1}$ such that the solution $w(t, x)$ of $(2.1)$ satisfies

$$
\int_{T / 4}^{3 T / 4} \int_{0}^{a_{1}} w(t, x)^{2} \mathrm{~d} x \mathrm{~d} t \leq \tilde{C}_{1} \int_{0}^{T} \int_{\omega_{1}} w(t, x)^{2} \mathrm{~d} x \mathrm{~d} t
$$

and

$$
\int_{T / 4}^{3 T / 4} \int_{b_{n}}^{a_{n+1}} w(t, x)^{2} \mathrm{~d} x \mathrm{~d} t \leq C_{n} \int_{0}^{T} \int_{\omega_{n}} w(t, x)^{2} \mathrm{~d} x \mathrm{~d} t+\tilde{C}_{n+1} \int_{0}^{T} \int_{\omega_{n+1}} w(t, x)^{2} \mathrm{~d} x \mathrm{~d} t .
$$

Moreover the constants $C_{n}$ and $C_{n+1}$ can be chosen as following:

$$
C_{n}:=K_{0} \mathrm{e}^{K_{0}\left(a_{n+1}-b_{n}\right)^{2}} \frac{1}{\min \left(1, b_{n}-a_{n}\right)^{4}},
$$

and

$$
\tilde{C}_{n+1}:=K_{0} \mathrm{e}^{K_{0}\left(a_{n+1}-b_{n}\right)^{2}} \frac{1}{\min \left(1, b_{n+1}-a_{n+1}\right)^{4}},
$$

where the constant $K_{0}$ is large enough and is independent of $n$. 
The constants $C_{n}$ and $\tilde{C}_{n+1}$ depend on the size of the domain and on the size of the control region. The interest of (3.3) and (3.4) is to give an estimate of this dependence when the size of the domain goes to infinity or the size of the control region goes to zero. It is not surprising to have constants that go to infinity when the size of the uncontrolled region goes to infinity, or when the size of the control region goes to zero.

Note that Lemma 3.1 easily implies Proposition 2.1: indeed, let $\rho_{2}$ be a function of class $\mathcal{C}^{1}$ on $\mathbb{R}_{+}$such that, for all $n, 1 / \rho_{2} \geq 1+C_{n}+\tilde{C}_{n}$ on $\omega_{n}$. Then we deduce from (3.2) that

$$
\int_{T / 4}^{3 T / 4} \int_{\Omega} w(t, x)^{2} \mathrm{~d} x \mathrm{~d} t \leq \sum_{n}\left(1+C_{n}+\tilde{C}_{n}\right) \int_{0}^{T} \int_{\omega_{n}} w(t, x)^{2} \mathrm{~d} x \mathrm{~d} t \leq \int_{0}^{T} \int_{\omega} w(t, x)^{2} \frac{\mathrm{d} x}{\rho_{2}(x)} \mathrm{d} t .
$$

The proof of (2.2) is similar, noting that (3.2) gives that

$$
\frac{1}{1+C_{n}+\tilde{C}_{n+1}} \int_{T / 4}^{3 T / 4} \int_{a_{n}}^{a_{n+1}} w(t, x)^{2} \mathrm{~d} x \mathrm{~d} t \leq \int_{0}^{T} \int_{\omega_{n} \cup \omega_{n+1}} w(t, x)^{2} \mathrm{~d} x \mathrm{~d} t .
$$

Hence it remains to prove Lemma 3.1.

Proof of Lemma 3.1. The proof of (3.1) and (3.2) are similar. We only prove (3.2).

Let $w(t, x)$ be the solution of (2.1). The proof of the observability inequality (3.2) follows from the global Carleman estimates for the heat equation on bounded domains with Dirichlet boundary conditions, used with adequate cut-off functions. We need to look closely to the different constants that appear, since we will need precise estimates in the following.

First we introduce some notations: for all $n$, we define

$$
\begin{aligned}
c_{n} & :=b_{n}-\frac{1}{2} \min \left(1, b_{n}-a_{n}\right), \\
c_{n+1}^{\prime} & :=a_{n+1}+\frac{1}{2} \min \left(1, b_{n+1}-a_{n+1}\right), \\
d_{n} & :=\frac{1}{2}\left(c_{n}+b_{n}\right),
\end{aligned}
$$

and

What is important is that:

$$
d_{n+1}^{\prime}:=\frac{1}{2}\left(a_{n+1}+c_{n+1}\right)
$$

$$
\begin{gathered}
a_{n}<c_{n}<d_{n}<b_{n}<a_{n+1}<d_{n+1}^{\prime}<c_{n+1}^{\prime}<b_{n+1}, \\
c_{n+1}^{\prime}-c_{n} \leq a_{n+1}-b_{n}+1,
\end{gathered}
$$

hence the domain $\left(c_{n}, c_{n+1}^{\prime}\right)$ has a size similar to the uncontrolled part $\left(b_{n}, a_{n+1}\right)$, and

$$
d_{n}-c_{n}=\frac{1}{4} \min \left(1, b_{n}-a_{n}\right), \quad c_{n+1}^{\prime}-d_{n+1}^{\prime}=\frac{1}{4} \min \left(1, b_{n+1}-a_{n+1}\right),
$$

hence the domains $\left(c_{n}, d_{n}\right)$ and $\left(d_{n+1}^{\prime}, c_{n+1}^{\prime}\right)$ have a size comparable to the control parts $\left(a_{n}, b_{n}\right)$ and $\left(a_{n+1}, b_{n+1}\right)$.

Now fix $n$ and construct a cut-off function $\psi$ such that $0 \leq \psi \leq 1$ on $\Omega, \psi=1$ on $\left(d_{n}, d_{n+1}^{\prime}\right)$ and $\psi=0$ outside $\left(c_{n}, c_{n+1}^{\prime}\right)$. (Of course $\psi$ depends on $n$.)

Let us now consider $z:=\psi w$. The function $z(t, x)$ satisfies the following heat equation in the bounded domain $\left(c_{n}, c_{n+1}^{\prime}\right)$ :

$$
\begin{cases}z_{t}+z_{x x}=h(t, x), & x \in\left(c_{n}, c_{n+1}\right), t \in(0, T), \\ z\left(t, c_{n}\right)=0=z\left(t, c_{n+1}^{\prime}\right), & t \in(0, T),\end{cases}
$$


where $h(t, x):=\left(\psi_{x} w\right)_{x}+\psi_{x} w_{x} \in L^{2}\left(\left(c_{n}, c_{n+1}^{\prime}\right) \times(0, T)\right)$. Note also that the function $h$ is supported in space in $\left(c_{n}, d_{n}\right) \cup\left(d_{n+1}^{\prime}, c_{n+1}^{\prime}\right)$. We denote $\lambda_{n}:=c_{n+1}^{\prime}-c_{n}$ the length of the interval $\left(c_{n}, c_{n+1}^{\prime}\right)$, and

$$
\theta(t):=\frac{1}{t(T-t)}, \quad \tilde{\sigma}_{n}(t, x):=\theta(t)\left(\mathrm{e}^{2}-\mathrm{e}^{\left(2 x-c_{n}-c_{n+1}^{\prime}\right) / \lambda_{n}}\right) .
$$

We prove the following

Lemma 3.2. There exists two positive constants $K\left(\lambda_{n}, T\right)$ and $R\left(\lambda_{n}, T\right)$ such that the solution $z$ of (3.6) satisfies the observability estimate:

$$
\int_{T / 4}^{3 T / 4} \int_{c_{n}}^{c_{n+1}^{\prime}} z(t, x)^{2} \mathrm{~d} x \mathrm{~d} t \leq K\left(\lambda_{n}, T\right) \int_{0}^{T} \int_{c_{n}}^{c_{n+1}^{\prime}} \mathrm{e}^{-2 R\left(\lambda_{n}, T\right) \tilde{\sigma}_{n}(t, x)} h(t, x)^{2} \mathrm{~d} x \mathrm{~d} t .
$$

Moreover the constants $K\left(\lambda_{n}, T\right)$ and $R\left(\lambda_{n}, T\right)$ can be chosen as following:

$$
\begin{gathered}
K\left(\lambda_{n}, T\right)=K_{1} \frac{\mathrm{e}^{K_{1} \max \left(\lambda_{n}, 1\right)^{2}}}{\max \left(\lambda_{n}, 1\right)^{2}}, \\
R\left(\lambda_{n}, T\right)=R_{1} \max \left(\lambda_{n}, 1\right)^{2},
\end{gathered}
$$

for some constants $K_{1}$ and $R_{1}$ large enough and independent of $n$.

Remark. Usual Carleman estimates (see, e.g., $[1,12,14])$ give that

$$
S\left\|\frac{\mathrm{e}^{-R \sigma}}{\sqrt{r}} z\right\|_{2, r}^{2} \leq K_{n}\left\|\mathrm{e}^{-R \sigma} h\right\|_{L^{2}\left((0, T) \times \mathcal{O}_{n}\right)}^{2}+K_{n} \int_{0}^{T} \int_{\partial \mathcal{O}_{n}} r \mathrm{e}^{-2 R \sigma} \partial_{\nu} \phi\left|\partial_{\nu} z\right|^{2},
$$

with the following notations: $\mathcal{O}_{n}:=\left(c_{n}, c_{n+1}^{\prime}\right)$,

$$
\|z\|_{2, r}^{2}:=\int_{0}^{T} \int_{\mathcal{O}_{n}}\left|r^{2} z\right|^{2}+\left|z_{t}\right|^{2}+\left|z_{x x}\right|^{2}+\left|r z_{x}\right|^{2} \mathrm{~d} x \mathrm{~d} t
$$

$\phi:\left(c_{n}, c_{n+1}^{\prime}\right) \rightarrow \mathbb{R}$ is a function of class $\mathcal{C}^{2}$ such that $\phi^{\prime}(x) \neq 0$ for all $x \in \mathcal{O}_{n}$, and

$$
\theta(t):=\frac{1}{t(T-t)}, \quad \sigma(t, x):=\theta(t)\left(\mathrm{e}^{2 S\|\phi\|_{\infty}}-\mathrm{e}^{S \phi(x)}\right), \quad r(t, x):=R S \theta(t) \mathrm{e}^{S \phi(x)},
$$

and finally $R, S$ and $K_{n}$ are constants that are large enough. Due to the cutoff function $\psi$, the term $\partial_{\nu} z$ is equal to zero on $\partial \mathcal{O}_{n}=\left\{c_{n}, c_{n+1}^{\prime}\right\}$. In Lemma 3.2 we specially study the dependence of the constants with respect to the size of the domain. Here we are only interested in studying the zero order term of the weighted norm (3.11), hence computations are a little shorter. To do them, we transform the heat equation in $z$ stated in the domain $\left(c_{n}, c_{n+1}^{\prime}\right)$ into a heat equation stated in the domain $(-1,1)$, with variable coefficients.

Let us admit Lemma 3.2 for the moment. The proof of Lemma 3.1 will need to estimate the right-hand side term of (3.7):

Lemma 3.3. For all $n$, there exists $K_{n}^{\prime}$ such that

$$
\int_{0}^{T} \int_{c_{n}}^{c_{n+1}^{\prime}} \mathrm{e}^{-2 R\left(\lambda_{n}, T\right) \tilde{\sigma}_{n}(t, x)} h(t, x)^{2} \mathrm{~d} x \mathrm{~d} t \leq K_{n}^{\prime} \int_{0}^{T} \int_{\omega_{n}} w^{2} \mathrm{~d} x \mathrm{~d} t+K_{n+1}^{\prime} \int_{0}^{T} \int_{\omega_{n+1}} w^{2} \mathrm{~d} x \mathrm{~d} t .
$$


Moreover the constant $K_{n}^{\prime}$ can be chosen as following:

$$
K_{n}^{\prime}=\frac{C_{0}}{\min \left(1, b_{n}-a_{n}\right)^{4}},
$$

where the constant $C_{0}$ is large enough and is independent of $n$.

Admitting Lemmas 3.2 and 3.3, we get that

$$
\begin{aligned}
\int_{T / 4}^{3 T / 4} \int_{b_{n}}^{a_{n+1}}|w|^{2} & \leq \int_{T / 4}^{3 T / 4} \int_{c_{n}}^{c_{n+1}^{\prime}} z(t, x)^{2} \mathrm{~d} x \mathrm{~d} t \\
& \leq K\left(\lambda_{n}, T\right) \int_{0}^{T} \int_{c_{n}}^{c_{n+1}^{\prime}} \mathrm{e}^{-2 R\left(\lambda_{n}, T\right) \tilde{\sigma}_{n}(t, x)} h(t, x)^{2} \mathrm{~d} x \mathrm{~d} t \\
& \leq K\left(\lambda_{n}, T\right) K_{n}^{\prime} \int_{0}^{T} \int_{\omega_{n}} w^{2} \mathrm{~d} x \mathrm{~d} t+K\left(\lambda_{n}, T\right) K_{n+1}^{\prime} \int_{0}^{T} \int_{\omega_{n+1}} w^{2} \mathrm{~d} x \mathrm{~d} t
\end{aligned}
$$

which gives (3.2), noting that, for some $K_{0}$ large enough, we have that

$$
K\left(\lambda_{n}, T\right) K_{n}^{\prime}=K_{1} \frac{\mathrm{e}^{K_{1} \max \left(\lambda_{n}, 1\right)^{2}}}{\max \left(\lambda_{n}, 1\right)^{2}} \frac{C_{0}}{\min \left(1, b_{n}-a_{n}\right)^{4}} \leq K_{0} \mathrm{e}^{K_{0}\left(a_{n+1}-b_{n}\right)^{2}} \frac{1}{\min \left(1, b_{n}-a_{n}\right)^{4}}=C_{n},
$$

and

$K\left(\lambda_{n}, T\right) K_{n+1}^{\prime}=K_{1} \frac{\mathrm{e}^{K_{1} \max \left(\lambda_{n}, 1\right)^{2}}}{\max \left(\lambda_{n}, 1\right)^{2}} \frac{C_{0}}{\min \left(1, b_{n+1}-a_{n+1}\right)^{4}} \leq K_{0} \mathrm{e}^{K_{0}\left(a_{n+1}-b_{n}\right)^{2}} \frac{1}{\min \left(1, b_{n+1}-a_{n+1}\right)^{4}}=\tilde{C}_{n+1}$.

Proof of Lemma 3.2. We do not detail too much the computations in this section, since we detail them for the $N$ dimensional case, see Section 5 . In the one dimensional case, computations are a little shorter.

First let us define the following change of variable

$$
y:=\frac{1}{\lambda_{n}}\left(2 x-c_{n}-c_{n+1}^{\prime}\right)
$$

and $Z(t, y):=z(t, x), H(t, y):=h(t, x)$. Then $Z$ satisfies

$$
\begin{cases}Z_{t}+\frac{4}{\lambda_{n}^{2}} Z_{y y}=H(t, y), & y \in(-1,1), t \in(0, T) \\ Z(t,-1)=0=Z(t, 1), & t \in(0, T)\end{cases}
$$

Take a strictly increasing function $\phi:[-1,1] \rightarrow \mathbb{R}$ of class $\mathcal{C}^{1}$, and define

$$
\alpha_{n}:=\frac{4}{\lambda_{n}^{2}}, \quad \theta(t):=\frac{1}{t(T-t)}, \quad \sigma(t, y):=\theta(t)\left(\mathrm{e}^{2 S\|\phi\|_{\infty}}-\mathrm{e}^{S \phi(y)}\right) .
$$

Now following [1] define

$$
\begin{aligned}
V(t, y) & :=\mathrm{e}^{-R \sigma(t, y)} Z(t, y), \\
P_{R}^{+} V: & =R \sigma_{t} V+R^{2} \alpha_{n} \sigma_{y}^{2} V+\alpha_{n} V_{y y} \\
P_{R}^{-} V & :=V_{t}+R \alpha_{n}\left(\sigma_{y} V\right)_{y}+R \alpha_{n} \sigma_{y} V_{y} .
\end{aligned}
$$


Then

$$
P_{R}^{+} V+P_{R}^{-} V=H \mathrm{e}^{-R \sigma},
$$

hence, denoting $\|\cdot\|$ and $\langle\cdot, \cdot\rangle$ the usual norm and scalar product of $L^{2}(-1,1)$,

$$
\left\|P_{R}^{+} V\right\|^{2}+\left\|P_{R}^{-} V\right\|^{2}+2\left\langle P_{R}^{+} V, P_{R}^{-} V\right\rangle=\left\|H \mathrm{e}^{-R \sigma}\right\|^{2} .
$$

On the other hand,

$$
\left\langle P_{R}^{+} V, P_{R}^{-} V\right\rangle=P_{1}+P_{2}+P_{3}+P_{4}
$$

where

$$
\begin{aligned}
& P_{1}=\left\langle R \sigma_{t} V+R^{2} \alpha_{n} \sigma_{y}^{2} V+\alpha_{n} V_{y y}, V_{t}\right\rangle, \\
& P_{2}=R^{2}\left\langle\sigma_{t} V, \alpha_{n}\left(\sigma_{y} V\right)_{y}+\alpha_{n} \sigma_{y} V_{y}\right\rangle, \\
& P_{3}=R^{3}\left\langle\alpha_{n} \sigma_{y}^{2} V, \alpha_{n}\left(\sigma_{y} V\right)_{y}+\alpha_{n} \sigma_{y} V_{y}\right\rangle, \\
& P_{4}=R\left\langle\alpha_{n} V_{y y}, \alpha_{n}\left(\sigma_{y} V\right)_{y}+\alpha_{n} \sigma_{y} V_{y}\right\rangle .
\end{aligned}
$$

Some integrations by part lead to the following identities:

$$
\begin{gathered}
P_{1}+P_{2}=-\int_{0}^{T} \int_{-1}^{1}\left(\frac{1}{2} R \sigma_{t t}+2 R^{2} \alpha_{n} \sigma_{y} \sigma_{y t}\right) V^{2} \mathrm{~d} y \mathrm{~d} t, \\
P_{3}=-R^{3} \int_{0}^{T} \int_{-1}^{1} 2 \alpha_{n}^{2} \sigma_{y}^{2} \sigma_{y y} V^{2} \mathrm{~d} y \mathrm{~d} t
\end{gathered}
$$

and

$$
\begin{aligned}
P_{4}=R \int_{0}^{T}\left[\alpha_{n}^{2} \sigma_{y} V_{y}^{2}\right]_{-1}^{1} \mathrm{~d} t-R \int_{0}^{T} \int_{-1}^{1} \alpha_{n}^{2} \sigma_{y y} V_{y}^{2} \mathrm{~d} y \mathrm{~d} t & \\
& +\left\langle P_{R}^{+} V, R \alpha_{n} \sigma_{y y} V\right\rangle-\int_{0}^{T} \int_{-1}^{1}\left(R^{2} \alpha_{n} \sigma_{t} \sigma_{y y}+R^{3} \alpha_{n}^{2} \sigma_{y}^{2} \sigma_{y y}\right) V^{2} \mathrm{~d} y \mathrm{~d} t .
\end{aligned}
$$

Since $V_{y}(t,-1)=0=V_{y}(t, 1)$, we obtain that

$$
\begin{aligned}
\left\langle P_{R}^{+} V, P_{R}^{-} V\right\rangle & \geq-R \int_{0}^{T} \int_{-1}^{1} \alpha_{n}^{2} \sigma_{y y} V_{y}^{2} \mathrm{~d} y \mathrm{~d} t-\frac{1}{2}\left\|P_{R}^{+} V\right\|^{2} \\
- & \int_{0}^{T} \int_{-1}^{1}\left(\frac{1}{2} R \sigma_{t t}+2 R^{2} \alpha_{n} \sigma_{y} \sigma_{y t}+2 R^{3} \alpha_{n}^{2} \sigma_{y}^{2} \sigma_{y y}+R^{2} \alpha_{n} \sigma_{t} \sigma_{y y}+R^{3} \alpha_{n}^{2} \sigma_{y}^{2} \sigma_{y y}+\frac{1}{2} R^{2} \alpha_{n}^{2} \sigma_{y y}^{2}\right) V^{2} \mathrm{~d} y \mathrm{~d} t .
\end{aligned}
$$

Let us now choose $\phi(y)=y$ and $S=1$. Then adding $(1 / 2)\left\|P_{R}^{-} V\right\|^{2}$ to the left-hand side of the previous inequality and noting that $-\sigma_{y y}=\theta \mathrm{e}^{y} \geq 0$, we obtain that

$$
\begin{array}{r}
\left\|P_{R} V\right\|^{2} \geq 2 \int_{0}^{T} \int_{-1}^{1}\left(-\frac{1}{2} R \sigma_{t t}-2 R^{2} \alpha_{n} \sigma_{y} \sigma_{y t}-2 R^{3} \alpha_{n}^{2} \sigma_{y}^{2} \sigma_{y y}\right. \\
\left.\quad-R^{2} \alpha_{n} \sigma_{t} \sigma_{y y}-R^{3} \alpha_{n}^{2} \sigma_{y}^{2} \sigma_{y y}-\frac{1}{2} R^{2} \alpha_{n}^{2} \sigma_{y y}^{2}\right) V^{2} \mathrm{~d} y \mathrm{~d} t .
\end{array}
$$

Now it is sufficient to study the coefficient of $V^{2}$ : choosing $R$ defined by (3.9):

$$
R:=R\left(\lambda_{n}, T\right)=R_{0} \max \left(\lambda_{n}, 1\right)^{2}
$$


and computing explicitly every term, it is easy to see that the term $-R^{3} \alpha_{n}^{2} \sigma_{y}^{2} \sigma_{y y}=R^{3} \alpha_{n}^{2} \theta^{3} \mathrm{e}^{3 y}$ can dominate the other terms if $R_{0}$ is chosen large enough. Thus we obtain that there is some $c_{T}$ such that

$$
\begin{aligned}
\int_{T / 4}^{3 T / 4} \int_{-1}^{1} Z(t, y)^{2} \mathrm{~d} y \mathrm{~d} t & \leq c_{T} \frac{\mathrm{e}^{2 R c_{T}}}{R^{3} \alpha_{n}^{2}} \int_{T / 4}^{3 T / 4} \int_{-1}^{1} R^{3} \alpha_{n}^{2} \sigma_{y}^{2}\left(-\sigma_{y y}\right) V^{2} \mathrm{~d} y \mathrm{~d} t \\
& \leq c_{T} \frac{\mathrm{e}^{2 R c_{T}}}{R^{3} \alpha_{n}^{2}}\left\|P_{R} V\right\|^{2}=c_{T} \frac{\mathrm{e}^{2 R c_{T}}}{R^{3} \alpha_{n}^{2}}\left\|H \mathrm{e}^{-R \sigma}\right\|^{2},
\end{aligned}
$$

and this gives (3.7) with (3.8).

Proof of Lemma 3.3. We will denote

$$
\|f\|_{\infty, I}:=\sup \{|f(x)|, x \in I\}
$$

Then using the definition of $h$, we deduce that

$$
\begin{aligned}
\int_{0}^{T} \int_{c_{n}}^{c_{n+1}^{\prime}} \mathrm{e}^{-2 R\left(\lambda_{n}, T\right) \tilde{\sigma}_{n}} h^{2} \mathrm{~d} x \mathrm{~d} t \leq & 8 \int_{0}^{T} \int_{c_{n}}^{c_{n+1}^{\prime}}\left(\psi_{x x}^{2} w^{2}+\psi_{x}^{2} w_{x}^{2}\right) \mathrm{e}^{-2 R\left(\lambda_{n}, T\right) \tilde{\sigma}_{n}} \mathrm{~d} x \mathrm{~d} t \\
\leq & 8\left\|\psi_{x x}\right\|_{\infty,\left(c_{n}, d_{n}\right)}^{2} \int_{0}^{T} \int_{\omega_{n}} w^{2} \mathrm{~d} x \mathrm{~d} t \\
& +8\left\|\psi_{x x}\right\|_{\infty,\left(d_{n+1}^{\prime}, c_{n+1}^{\prime}\right)}^{2} \int_{0}^{T} \int_{\omega_{n+1}} w^{2} \mathrm{~d} x \mathrm{~d} t \\
& +8\left\|\psi_{x}\right\|_{\infty,\left(c_{n}, d_{n}\right)}^{2} \int_{0}^{T} \int_{c_{n}}^{d_{n}} w_{x}^{2} \mathrm{e}^{-2 R\left(\lambda_{n}, T\right) \tilde{\sigma}_{n}} \mathrm{~d} x \mathrm{~d} t \\
& +8\left\|\psi_{x}\right\|_{\infty,\left(d_{n+1}^{\prime}, c_{n+1}^{\prime}\right)}^{2} \int_{0}^{T} \int_{d_{n+1}^{\prime}}^{c_{n+1}^{\prime}} w_{x}^{2} \mathrm{e}^{-2 R\left(\lambda_{n}, T\right) \tilde{\sigma}_{n}} \mathrm{~d} x \mathrm{~d} t .
\end{aligned}
$$

The first two terms of the right-hand side of (3.16) are exactly what we want (see the right-hand side of (3.2)). It remains to estimate the last two terms of (3.16). We estimate the first one, the second is similar. This is a classical Caccioppoli-type inequality: we introduce a second cut-off function $\xi$, such that $0 \leq \xi \leq 1, \xi=1$ on $\left(c_{n}, d_{n}\right)$, and $\xi=0$ outside $\left(a_{n}, b_{n}\right)$. Define $\eta:=\xi \mathrm{e}^{-R\left(\lambda_{n}, T\right) \tilde{\sigma}_{n}}$. By integration by parts, we easily verify that

$$
\frac{1}{2} \frac{\mathrm{d}}{\mathrm{d} t} \int_{a_{n}}^{b_{n}} \eta^{2} w^{2} \mathrm{~d} x=\int_{a_{n}}^{b_{n}} \eta \eta_{t} w^{2}+\eta^{2} w_{x}^{2}+2 \eta \eta_{x} w_{x} w \mathrm{~d} x
$$

Hence using that $\eta(t=0) \equiv 0 \equiv \eta(t=T)$,

$$
\begin{aligned}
\int_{0}^{T} \int_{a_{n}}^{b_{n}} \eta^{2} w_{x}^{2}= & \frac{1}{2} \int_{a_{n}}^{b_{n}} \eta(T, x)^{2} w(T, x)^{2} \mathrm{~d} x \\
& -\frac{1}{2} \int_{a_{n}}^{b_{n}} \eta(0, x)^{2} w(0, x)^{2} \mathrm{~d} x-\int_{0}^{T} \int_{a_{n}}^{b_{n}} \eta \eta_{t} w^{2}+2 \eta \eta_{x} w_{x} w \mathrm{~d} x \mathrm{~d} t \\
= & -\int_{0}^{T} \int_{a_{n}}^{b_{n}} \eta \eta_{t} w^{2}+2 \eta \eta_{x} w_{x} w \mathrm{~d} x \mathrm{~d} t \\
\leq & \left\|\eta \eta_{t}\right\|_{\infty,\left(a_{n}, b_{n}\right) \times(0, T)} \int_{0}^{T} \int_{a_{n}}^{b_{n}} w^{2}+\frac{1}{2} \int_{0}^{T} \int_{a_{n}}^{b_{n}} \eta^{2} w_{x}^{2}+2 \int_{0}^{T} \int_{a_{n}}^{b_{n}} \eta_{x}^{2} w^{2}
\end{aligned}
$$


Hence

$$
\int_{0}^{T} \int_{c_{n}}^{d_{n}} w_{x}^{2} \mathrm{e}^{-2 R\left(\lambda_{n}, T\right) \tilde{\sigma}_{n}} \mathrm{~d} x \mathrm{~d} t \leq 4\left(\left\|\eta \eta_{t}\right\|_{\infty, \omega_{n} \times(0, T)}+\left\|\eta_{x}\right\|_{\infty, \omega_{n} \times(0, T)}^{2}\right) \int_{0}^{T} \int_{\omega_{n}} w^{2} .
$$

Then (3.12) follows from (3.16) and (3.17) with

$$
K_{n}^{\prime}:=8\left\|\psi_{x x}\right\|_{\infty, \omega_{n}}^{2}+32\left\|\psi_{x}\right\|_{\infty, \omega_{n}}^{2}\left(\left\|\eta \eta_{t}\right\|_{\infty, \omega_{n} \times(0, T)}+\left\|\eta_{x}\right\|_{\infty, \omega_{n} \times(0, T)}^{2}\right)
$$

Now let us estimate $K_{n}^{\prime}$. We can choose the cut-off functions such that, for some constants $c$ independent of $n$, we have

$$
\begin{gathered}
\left\|\psi_{x}\right\|_{\infty, \omega_{n}}=\frac{c}{d_{n}-c_{n}}, \\
\left\|\psi_{x x}\right\|_{\infty, \omega_{n}}=\frac{c}{\left(d_{n}-c_{n}\right)^{2}}, \\
\|\eta\|_{\infty, \omega_{n} \times(0, T) \leq 1} \\
\left\|\eta_{t}\right\|_{\infty, \omega_{n} \times(0, T)} \leq c \\
\left\|\xi_{x} \mathrm{e}^{-R\left(\lambda_{n}, T\right) \tilde{\sigma}_{n}}\right\|_{\infty, \omega_{n} \times(0, T)} \leq\left\|\xi_{x}\right\|_{\infty, \omega_{n}}=\frac{c}{d_{n}-c_{n}}, \\
\left\|\xi R\left(\lambda_{n}, T\right){\tilde{\sigma_{n x}}} \mathrm{e}^{-R\left(\lambda_{n}, T\right) \tilde{\sigma}_{n}}\right\|_{\infty, \omega_{n} \times(0, T)} \leq c, \\
\left\|\eta_{x}\right\|_{\infty, \omega_{n} \times(0, T)}=\frac{c}{d_{n}-c_{n}} .
\end{gathered}
$$

Hence

$$
\frac{C_{0}}{\min \left(1, b_{n}-a_{n}\right)^{4}}
$$

is a suitable upper bound for $K_{n}^{\prime}$. This confirms $(3.13)$.

\subsection{Proof of part (i) of Theorem 2.1}

Part (i) of Theorem 2.1 follows from the observability inequality (2.2). We recall that given $u_{0} \in L^{2}(\Omega)$ and $f \in L^{2}(\Omega \times(0, T))$, the problem $(1.2)$ has one and only one solution $u^{f}(t, x) \in \mathcal{C}^{0}\left([0, T] ; L^{2}(\Omega)\right) \cap L^{2}\left(0, T ; H_{0}^{1}(\Omega)\right)$.

Fix $\varepsilon>0$ and consider as usual the functional

$$
J_{\varepsilon}(f):=\frac{1}{2} \int_{0}^{T} \int_{\Omega}|f|^{2} \mathrm{~d} x \mathrm{~d} t+\frac{1}{2 \varepsilon} \int_{\Omega}\left|u^{f}(T)\right|^{2} \mathrm{~d} x, \quad f \in L^{2}(\Omega \times(0, T)) .
$$

It is well known that the functional $J_{\varepsilon}$ is well defined, strictly convex, and $J_{\varepsilon}(f) \rightarrow \infty$ as $\|f\|_{L^{2}(\Omega \times(0, T))} \rightarrow \infty$. It attains its minimum at $f^{\varepsilon} \in L^{2}(\Omega \times(0, T))$ characterized by:

$$
f^{\varepsilon}(t, x)=-v^{\varepsilon}(t, x) \chi_{\omega}(x)
$$

where $v^{\varepsilon}$ is the solution of the adjoint problem

$$
\begin{cases}v_{t}^{\varepsilon}+v_{x x}^{\varepsilon}=0, & x \in \Omega, t \in(0, T), \\ v^{\varepsilon}(t, x=0)=0, & t \in(0, T) \\ v^{\varepsilon}(t=T, x)=\frac{1}{\varepsilon} u^{f^{\varepsilon}}(T) \in L^{2}(\Omega) . & \end{cases}
$$


Now we need suitable $a$ priori estimates to let $\varepsilon \rightarrow 0$. We multiply (1.2) by $v^{\varepsilon}$ and (3.20) by $u^{f^{\varepsilon}}$, we add these identities, we integrate on $\Omega \times(0, T)$, and we obtain:

$$
\int_{0}^{T} \int_{\Omega} u_{t}^{f^{\varepsilon}} v^{\varepsilon}+u^{f^{\varepsilon}} v_{t}^{\varepsilon} \mathrm{d} x \mathrm{~d} t=\int_{0}^{T} \int_{\Omega} f^{\varepsilon} v^{\varepsilon} \mathrm{d} x \mathrm{~d} t
$$

hence

$$
\frac{1}{\varepsilon} \int_{\Omega}\left|u^{f^{\varepsilon}}(T, x)\right|^{2} \mathrm{~d} x+\int_{0}^{T} \int_{\Omega}\left|f^{\varepsilon}(t, x)\right|^{2} \mathrm{~d} x \mathrm{~d} t=\int_{\Omega} u_{0}(x) v^{\varepsilon}(0, x) \mathrm{d} x .
$$

Now we need to estimate $v^{\varepsilon}(0, x)$. Let $w$ be the solution of (2.1) and consider the function

$$
N: t \in[0, T] \mapsto \int_{\Omega}|w(t, x)|^{2} \rho_{1}(x) \mathrm{d} x .
$$

Then

$$
N^{\prime}(t)=2 \int_{\Omega} w w_{t} \rho_{1} \mathrm{~d} x=-2 \int_{\Omega} w w_{x x} \rho_{1} \mathrm{~d} x=2 \int_{\Omega}\left|w_{x}\right|^{2} \rho_{1}+w w_{x} \rho_{1, x} \mathrm{~d} x .
$$

When $\rho_{1}$ satisfy the assumption (2.5): $\left|\rho_{1, x}\right| \leq \alpha_{1} \rho_{1}$, we have

$$
\begin{aligned}
& N^{\prime}(t) \geq 2 \int_{\Omega}\left|w_{x}\right|^{2} \rho_{1} \mathrm{~d} x-2 \alpha_{1} \int_{\Omega}\left|w w_{x}\right| \rho_{1} \mathrm{~d} x \\
& \quad \geq 2 \int_{\Omega}\left|w_{x}\right|^{2} \rho_{1} \mathrm{~d} x-2 \int_{\Omega}\left|w_{x}\right|^{2} \rho_{1} \mathrm{~d} x-\frac{\alpha_{1}^{2}}{2} \int_{\Omega}|w|^{2} \rho_{1} \mathrm{~d} x=-\frac{\alpha_{1}^{2}}{2} N(t) .
\end{aligned}
$$

Hence

$$
\forall t \in(0, T), N(t) \geq N(0) \mathrm{e}^{-\alpha_{1}^{2} t / 2} .
$$

Combining this estimate with the observability inequality $(2.2)$, we obtain the following observability inequality:

$$
\begin{aligned}
N(0)=\int_{\Omega}|w(0, x)|^{2} \rho_{1}(x) \mathrm{d} x \leq \frac{2}{T} \mathrm{e}^{3 \alpha_{1}^{2} T / 8} \int_{T / 4}^{3 T / 4} \int_{\Omega}|w(t, x)|^{2} \rho_{1}(x) \mathrm{d} x \mathrm{~d} t & \\
& \leq \frac{2}{T} \mathrm{e}^{3 \alpha_{1}^{2} T / 8} \int_{0}^{T} \int_{\omega}|w(t, x)|^{2} \mathrm{~d} x \mathrm{~d} t .
\end{aligned}
$$

Now we can estimate the right-hand side of (3.21): we deduce from (3.22) that

$$
\begin{aligned}
\int_{\Omega} u_{0}(x) v^{\varepsilon}(0, x) \mathrm{d} x & \leq\left(\int_{\Omega}\left|u_{0}\right|^{2} \frac{\mathrm{d} x}{\rho_{1}(x)}\right)^{1 / 2}\left(\int_{\Omega}\left|v^{\varepsilon}(0, x)\right|^{2} \rho_{1}(x) \mathrm{d} x\right)^{1 / 2} \\
& \leq\left(\int_{\Omega}\left|u_{0}\right|^{2} \frac{\mathrm{d} x}{\rho_{1}(x)}\right)^{1 / 2}\left(\frac{2}{T} \mathrm{e}^{3 \alpha_{1}^{2} T / 8} \int_{0}^{T} \int_{\omega}\left|v^{\varepsilon}(t, x)\right|^{2} \mathrm{~d} x \mathrm{~d} t\right)^{1 / 2} \\
& \leq C_{T} \int_{\Omega}\left|u_{0}\right|^{2} \frac{\mathrm{d} x}{\rho_{1}(x)}+\frac{1}{2} \int_{0}^{T} \int_{\omega}\left|v^{\varepsilon}(t, x)\right|^{2} \mathrm{~d} x \mathrm{~d} t .
\end{aligned}
$$

Since $f^{\varepsilon}=-v^{\varepsilon}$ on $\omega \times(0, T)$, we obtain from (3.21) that

$$
\frac{1}{\varepsilon} \int_{\Omega}\left|u^{f^{\varepsilon}}(T, x)\right|^{2} \mathrm{~d} x+\frac{1}{2} \int_{0}^{T} \int_{\omega}\left|f^{\varepsilon}(t, x)\right|^{2} \mathrm{~d} x \mathrm{~d} t \leq C_{T} \int_{\Omega}\left|u_{0}\right|^{2} \frac{\mathrm{d} x}{\rho_{1}(x)}
$$


When $u_{0} \rho_{1}^{-1 / 2} \in L^{2}(\Omega)$, the sequences $\left(f^{\varepsilon}\right)_{\varepsilon}$ and $\left(\varepsilon^{-1 / 2} u^{f^{\varepsilon}}(T)\right)_{\varepsilon}$ are bounded respectively in $L^{2}(\omega \times(0, T))$ and $L^{2}(\Omega)$. Then we can extract from $\left(f^{\varepsilon}\right)_{\varepsilon}$ a subsequence that converges weakly in $L^{2}(\omega \times(0, T))$ to some limit $f^{0}$ such that the solution of (1.2) with $f=f^{0}$ satisfies $u^{f^{0}}(T)=0$.

\subsection{Proof of part (ii) of Theorem 2.1}

Part (ii) of Theorem 2.1 follows from the observability inequality (2.3).

Denote $\tilde{A}$ the unbounded operator $\frac{\mathrm{d}^{2}}{\mathrm{~d} x^{2}}$ with the Dirichlet boundary condition $u(x=0)=0$. The domain of $\tilde{A}$ is

$$
D(\tilde{A}):=\left\{u \in H_{\rho_{2}}^{2}(\Omega) \text { s.t. } u(x=0)=0\right\} .
$$

Denote also $\tilde{B}: L_{\rho_{2}}^{2}(\Omega) \rightarrow L_{\rho_{2}}^{2}(\Omega), \tilde{B}(f):=f \chi_{\omega}$. Now the problem (1.2) can be recast into the abstract form

$$
\left\{\begin{array}{l}
u_{t}=\tilde{A} u+\tilde{B} f \\
u(0)=u_{0}
\end{array}\right.
$$

Standard arguments can be used to justify that, if $\rho_{2}$ satisfies the differential inequality $\left|\rho_{2, x}\right| \leq \alpha \rho_{2}$, then the operator $\tilde{A}$ is the generator of an analytic semi-group in $L_{\rho_{2}}^{2}(\Omega)$ (see appendix for the proof). Therefore, given $u_{0} \in H_{\rho_{2}}^{1}(\Omega)$ and $f \in L^{2}\left((0, T) ; L_{\rho_{2}}^{2}(\Omega)\right)$, the problem (1.2) has one and only one solution $u^{f}(t, x) \in$ $L^{2}(0, T ; D(\tilde{A})) \cap H^{1}\left(0, T ; L_{\rho_{2}}^{2}(\Omega)\right)$.

Now we can study the controllability problem. Fix $\varepsilon>0$ and consider the weighted functional

$$
\tilde{J}_{\varepsilon}(f):=\frac{1}{2} \int_{0}^{T} \int_{\Omega}|f|^{2} \rho_{2} \mathrm{~d} x \mathrm{~d} t+\frac{1}{2 \varepsilon} \int_{\Omega}\left|u^{f}(T)\right|^{2} \rho_{2} \mathrm{~d} x .
$$

The functional $\tilde{J}_{\varepsilon}$ is well defined, strictly convex, and $\tilde{J}_{\varepsilon}(f) \rightarrow \infty$ as $\|f\|_{L^{2}\left((0, T) ; L_{\rho_{2}}^{2}(\Omega)\right)} \rightarrow \infty$. It attains its minimum in $\tilde{f}^{\varepsilon} \in L^{2}\left((0, T) ; L_{\rho_{2}}^{2}(\Omega)\right)$ characterized by: $D \tilde{J}_{\varepsilon}\left(\tilde{f}^{\varepsilon}\right) \cdot h=0$ for all $h \in L^{2}\left((0, T) ; L_{\rho_{2}}^{2}(\Omega)\right)$. (As usual, $D \tilde{J}_{\varepsilon}\left(\tilde{f}^{\varepsilon}\right) \cdot h=0$ denotes the differential of the functional $\tilde{J}_{\varepsilon}$ computed at the point $\tilde{f}^{\varepsilon}$ and applied to the element $h$.) Some care has to be taken here because we work in weighted spaces, and that will bring some changes to the usual results that we used in the previous subsection.

In order to compute $D \tilde{J}_{\varepsilon}\left(\tilde{f}^{\varepsilon}\right) \cdot h$, we consider the problem into its abstract form (3.24). The solution of $(3.24)$ is

$$
u^{f}(t)=\mathrm{e}^{t \tilde{A}} u_{0}+\int_{0}^{t} \mathrm{e}^{(t-s) \tilde{A}} \tilde{B} f(s) \mathrm{d} s=: \mathrm{e}^{t \tilde{A}} u_{0}+\tilde{L_{t}} f .
$$

Now we can compute $D \tilde{J}_{\varepsilon}(f) \cdot h$ :

$$
\tilde{J}_{\varepsilon}(f+h)-\tilde{J}_{\varepsilon}(f)=\frac{1}{2} \int_{0}^{T} \int_{\Omega}\left(2 f h+|h|^{2}\right) \rho_{2} \mathrm{~d} x \mathrm{~d} t+\frac{1}{2 \varepsilon} \int_{\Omega}\left(\left|u^{f+h}(T)\right|^{2}-\left|u^{f}(T)\right|^{2}\right) \rho_{2} \mathrm{~d} x .
$$

Note that

where $v^{h}$ is the solution of

$$
u^{f+h}=u^{f}+v^{h}
$$

$$
\left\{\begin{array}{l}
v_{t}^{h}=\tilde{A} v^{h}+\tilde{B} h \\
v^{h}(0)=0
\end{array}\right.
$$

Hence $v^{h}(T)=\tilde{L_{T}} h$. Therefore

$$
\tilde{J}_{\varepsilon}(f+h)-\tilde{J}_{\varepsilon}(f)=\frac{1}{2} \int_{0}^{T} \int_{\Omega}\left(2 f h+|h|^{2}\right) \rho_{2} \mathrm{~d} x \mathrm{~d} t+\frac{1}{2 \varepsilon} \int_{\Omega}\left(2 u^{f}(T) v^{h}(T)+\left|v^{h}(T)\right|^{2}\right) \rho_{2} \mathrm{~d} x .
$$


We note that (3.27) gives that

$$
\int_{\Omega} v_{t}^{h} v^{h} \rho_{2}+\int_{\Omega} v_{x}^{h} v_{x}^{h} \rho_{2}+v_{x}^{h} v^{h} \rho_{2, x}=\int_{\omega} h v^{h} \rho_{2} .
$$

Hence

$$
\begin{aligned}
\frac{1}{2}\left\|v^{h}(t)\right\|_{\rho_{2}}^{2} & =\int_{0}^{t} \int_{\omega} h v^{h} \rho_{2}-\int_{0}^{t} \int_{\Omega}\left|v_{x}^{h}\right|^{2} \rho_{2}+v_{x}^{h} v^{h} \rho_{2, x} \\
& \leq \int_{0}^{t} \int_{\Omega}\left|h v^{h}\right| \rho_{2}-\left|v_{x}^{h}\right|^{2} \rho_{2}+\left|v_{x}^{h}\right|^{2} \rho_{2}+\frac{\alpha_{2}^{2}}{4}\left|v^{h}\right|^{2} \rho_{2} \\
& \leq \int_{0}^{t} \frac{1}{2}\|h(t)\|_{\rho_{2}}^{2}+\frac{2+\alpha_{2}^{2}}{4}\left\|v^{h}(t)\right\|_{\rho_{2}}^{2} \\
& \leq \frac{1}{2}\|h\|_{L^{2}\left((0, T) ; L_{\rho_{2}}^{2}(\Omega)\right)}^{2}+\frac{2+\alpha_{2}^{2}}{4} \int_{0}^{t}\left\|v^{h}(t)\right\|_{\rho_{2}}^{2} \mathrm{~d} t .
\end{aligned}
$$

Then the Gronwall inequality gives that

$$
\left\|v^{h}(t)\right\|_{\rho_{2}}^{2} \leq\|h\|_{L^{2}\left((0, T) ; L_{\rho_{2}}^{2}(\Omega)\right)}^{2} \mathrm{e}^{\left(2+\alpha_{2}^{2}\right) t / 2}
$$

Thus

$$
D \tilde{J}_{\varepsilon}(f) \cdot h=\int_{0}^{T} \int_{\Omega} f h \rho_{2} \mathrm{~d} x \mathrm{~d} t+\frac{1}{\varepsilon} \int_{\Omega} u^{f}(T) v^{h}(T) \rho_{2} \mathrm{~d} x .
$$

Now we look closer to the last term of (3.28):

$$
\begin{aligned}
\int_{\Omega} u^{f}(T) v^{h}(T) \rho_{2} \mathrm{~d} x & =\left\langle u^{f}(T), v^{h}(T)\right\rangle_{\rho_{2}} \\
& =\left\langle u^{f}(T), \tilde{L_{T}} h\right\rangle_{\rho_{2}} \\
& =\left\langle u^{f}(T), \int_{0}^{T} \mathrm{e}^{(T-s) \tilde{A}} \tilde{B} h(s) \mathrm{d} s\right\rangle_{\rho_{2}} \\
& =\int_{0}^{T}\left\langle u^{f}(T), \mathrm{e}^{(T-s) \tilde{A}} \tilde{B} h(s)\right\rangle_{\rho_{2}} \mathrm{~d} s \\
& =\int_{0}^{T}\left\langle\tilde{B}^{*} \mathrm{e}^{(T-s) \tilde{A}^{*}} u^{f}(T), h(s)\right\rangle_{\rho_{2}} \mathrm{~d} s .
\end{aligned}
$$

Hence $D \tilde{J}_{\varepsilon}(f) \cdot h=0$ for all $h \in L^{2}\left((0, T) ; L_{\rho_{2}}^{2}(\Omega)\right)$ if and only if

$$
f+\frac{1}{\varepsilon} \tilde{B}^{*} \mathrm{e}^{(T-s) \tilde{A}^{*}} u^{f}(T)=0 .
$$

Thus $\tilde{J}_{\varepsilon}$ reaches its minimum in $\tilde{f}^{\varepsilon}$ characterized by

$$
\tilde{f}^{\varepsilon}=-\frac{1}{\varepsilon} \tilde{B}^{*} \mathrm{e}^{(T-s) \tilde{A}^{*}} u^{\tilde{f}^{\varepsilon}}(T) .
$$

We have now to understand what is the operator $\tilde{B}^{*} \mathrm{e}^{(T-s) \tilde{A}^{*}}$. First, the operator $\tilde{B}: L_{\rho_{2}}^{2}(\Omega) \rightarrow L_{\rho_{2}}^{2}(\Omega)$ is self-adjoint. Now for $\tilde{v_{0}} \in L_{\rho_{2}}^{2}(\Omega)$, denote

$$
\tilde{v}(s):=\mathrm{e}^{(T-s) \tilde{A}^{*}} \tilde{v_{0}}
$$


$\tilde{v}$ is the solution of the problem

$$
\left\{\begin{array}{l}
\tilde{v}_{s}+\tilde{A}^{*} \tilde{v}=0 \\
\tilde{v}(T)=\tilde{v_{0}}
\end{array}\right.
$$

Hence it remains to understand what is the operator $\tilde{A}^{*}$. Fix $\tilde{k} \in L_{\rho_{2}}^{2}(\Omega)$ : then

$$
\left\langle\tilde{A}^{*} \tilde{v_{0}}, \tilde{k}\right\rangle_{\rho_{2}}=\left\langle\tilde{v_{0}}, \tilde{A} \tilde{k}\right\rangle_{\rho_{2}}=\int_{\Omega} \tilde{v_{0}} \tilde{k}_{x x} \rho_{2} \mathrm{~d} x=\int_{\Omega}\left(\tilde{v_{0}} \rho_{2}\right)_{x x} \tilde{k} \mathrm{~d} x=\left\langle\frac{1}{\rho_{2}}\left(\tilde{v_{0}} \rho_{2}\right)_{x x}, \tilde{k}\right\rangle_{\rho_{2}} .
$$

Thus we obtain that $\tilde{J}_{\varepsilon}$ reaches its minimum at $\tilde{f}^{\varepsilon}$ characterized by

$$
\tilde{f}^{\varepsilon}(t, x)=-\tilde{v}^{\varepsilon}(t, x) \chi_{\omega}(x),
$$

where $\tilde{v}^{\varepsilon}$ is the solution of the adjoint problem

$$
\begin{cases}\tilde{v}_{t}^{\varepsilon}+\tilde{A}^{*} \tilde{v}^{\varepsilon}=\tilde{v}_{t}^{\varepsilon}+\frac{1}{\rho_{2}}\left(\tilde{v}^{\varepsilon} \rho_{2}\right)_{x x}=0, & x \in \Omega, t \in(0, T), \\ \tilde{v}^{\varepsilon}(t, x=0)=0, & t \in(0, T), \\ \tilde{v}^{\varepsilon}(t=T, x)=\frac{1}{\varepsilon} u^{\tilde{f}^{\varepsilon}}(T) \in L_{\rho_{2}}^{2}(\Omega) . & \end{cases}
$$

Note that

is solution of

$$
w(t, x):=\tilde{v}^{\varepsilon}(t, x) \rho_{2}(x)
$$

$$
\begin{cases}w_{s}+w_{x x}=0, & x \in \Omega, t \in(0, T), \\ w(t, x=0)=0, & t \in(0, T) \\ w(T, x)=\frac{1}{\varepsilon} u \tilde{f}^{\varepsilon}(T) \rho_{2}(x) \in L^{2}(\Omega) . & \end{cases}
$$

Now we need suitable a-priori estimates to let $\varepsilon \rightarrow 0$. We multiply (1.2) by $\tilde{v}^{\varepsilon} \rho_{2}$ and (3.32) by $u^{\tilde{f}^{\varepsilon}} \rho_{2}$, we add these identities, and we obtain:

$$
\left\langle u_{t}^{\tilde{f}^{\varepsilon}}, \tilde{v}^{\varepsilon}\right\rangle_{\rho_{2}}+\left\langle u^{\tilde{f}^{\varepsilon}}, \tilde{v}_{t}^{\varepsilon}\right\rangle_{\rho_{2}}=\left\langle\tilde{f}^{\varepsilon}, \tilde{v}^{\varepsilon}\right\rangle_{\rho_{2}} .
$$

Integrating on $(0, T)$ leads to

$$
\frac{1}{\varepsilon} \int_{\Omega}\left|u^{\tilde{f}^{\varepsilon}}(T, x)\right|^{2} \rho_{2}(x) \mathrm{d} x+\int_{0}^{T} \int_{\Omega}\left|\tilde{f}^{\varepsilon}(t, x)\right|^{2} \rho_{2}(x) \mathrm{d} x \mathrm{~d} t=\int_{\Omega} u_{0}(x) \tilde{v}^{\varepsilon}(0, x) \rho_{2}(x) \mathrm{d} x .
$$

Now we need to estimate $v^{\varepsilon}(0, x) \rho_{2}(x)$ : using (3.33), (3.34) and (2.3), we see that

$$
\begin{aligned}
\int_{T / 4}^{3 T / 4} \int_{\Omega} w(t, x)^{2} \mathrm{~d} x & \leq \int_{0}^{T} \int_{\omega} w(t, x)^{2} \frac{\mathrm{d} x}{\rho_{2}(x)} \mathrm{d} t \\
& =\int_{0}^{T} \int_{\omega} \tilde{v}^{\varepsilon}(t, x)^{2} \rho_{2}(x) \mathrm{d} x \mathrm{~d} t \\
& =\int_{0}^{T} \int_{\Omega} \tilde{f}^{\varepsilon}(t, x)^{2} \rho_{2}(x) \mathrm{d} x \mathrm{~d} t .
\end{aligned}
$$

Now just note that for the solution $w$ of (3.34) we have for all $t \in[0, T]$

$$
\int_{\Omega} w(0, x)^{2} \mathrm{~d} x \leq \int_{\Omega} w(t, x)^{2} \mathrm{~d} x
$$


hence

$$
\int_{\Omega} w(0, x)^{2} \mathrm{~d} x \leq \frac{2}{T} \int_{T / 4}^{3 T / 4} \int_{\Omega} w(t, x)^{2} \mathrm{~d} x
$$

Thus (3.36) implies that

$$
\int_{\Omega}\left|\tilde{v}^{\varepsilon}(0, x) \rho_{2}(x)\right|^{2} \mathrm{~d} x \leq \frac{2}{T} \int_{0}^{T} \int_{\Omega} \tilde{f}^{\varepsilon}(t, x)^{2} \rho_{2}(x) \mathrm{d} x \mathrm{~d} t .
$$

This estimate allows us to conclude the proof: using the Cauchy-Schwarz inequality, (3.35) and (3.37) give that

$$
\frac{1}{\varepsilon} \int_{\Omega}\left|u^{\tilde{f}^{\varepsilon}}(T, x)\right|^{2} \rho_{2}(x) \mathrm{d} x+\frac{1}{2} \int_{0}^{T} \int_{\Omega}\left|\tilde{f}^{\varepsilon}(t, x)\right|^{2} \rho_{2}(x) \mathrm{d} x \mathrm{~d} t \leq C_{T} \int_{\Omega}\left|u_{0}(x)\right|^{2} \mathrm{~d} x .
$$

When $u_{0} \in L^{2}(\Omega)$, the sequences $\left(\tilde{f}^{\varepsilon} \chi_{\omega}\right)_{\varepsilon}$ and $\left(\varepsilon^{-1 / 2} u^{\tilde{f}^{\varepsilon}}(T)\right)_{\varepsilon}$ are bounded respectively in $L^{2}\left((0, T) ; L_{\rho_{2}}^{2}(\Omega)\right)$ and $L_{\rho_{2}}^{2}(\Omega)$. Therefore there exists $f^{0} \in L^{2}\left((0, T) ; L_{\rho_{2}}^{2}(\Omega)\right)$ such that the solution of $(1.2)$ satisfies $u^{f^{0}}(T)=0$ in $L_{\rho_{2}}^{2}(\Omega)$.

\section{Estimates on the Weight FUnCtions}

As we have seen, for both part (i) and (ii) of Theorem 2.1, it is essential that both functions $\rho_{1}$ and $\rho_{2}$ satisfy the same differential inequality, that says, roughly speaking that $\rho_{1}$ and $\rho_{2}$ do not decay to 0 at infinity faster than some exponential function $\mathrm{e}^{-c x}$. To study the behavior of $\rho_{1}$ and $\rho_{2}$ at infinity, we study the behavior of the constants $C_{n}$ and $\tilde{C}_{n+1}$ that appear in (3.2) as $n \rightarrow \infty$, for which we have an explicit expression. We look for some conditions on the sequences $\left(a_{n}\right)_{n}$ and $\left(b_{n}\right)_{n}$ insuring that

$$
1+C_{n}+\tilde{C}_{n} \leq R \mathrm{e}^{R a_{n}}
$$

for all $n$ and some $R$ large enough. If (4.1) is satisfied, then

$$
\rho_{2}(x):=\frac{1}{R} \mathrm{e}^{-R x}
$$

satisfies

$$
1+C_{n}+\tilde{C}_{n} \leq R \mathrm{e}^{R a_{n}} \leq R \mathrm{e}^{R x} \leq \frac{1}{\rho_{2}(x)}
$$

for all $x \in \omega_{n}$. Then we deduce from (3.5) that inequality (2.3) will be satisfied, with a function $\rho_{2}$ satisfying (2.5). At last, we note from (3.3) and (3.4) that (4.1) is satisfied if

$$
\left\{\begin{array}{l}
\left(a_{n+1}-b_{n}\right)^{2} \leq r a_{n} \\
\left|\omega_{n}\right| \geq r^{-1} \mathrm{e}^{-r a_{n}}
\end{array}\right.
$$

for some constant $r$.

\subsection{Example 1}

The first interesting case is when the lengths of the uncontrolled parts are bounded above by some positive constant $M$, and the controlled parts are bounded below by some $m>0$ :

$$
a_{n+1}-b_{n} \leq M, \quad b_{n}-a_{n} \geq m .
$$


In that case, $C_{n}$ and $\tilde{C}_{n}$ are constants independent of $n$ (see (3.3) and (3.4)). Hence $\rho_{1}$ and $\rho_{2}$ are also constant functions, hence the weighted spaces are in fact the usual (non weighted) ones, and we have the null controllability of (1.2).

\subsection{Example 2}

It is more interesting to study what happens when $\left|\omega_{n}\right| \rightarrow 0$ as $n \rightarrow \infty$. In that case

$$
C_{n}=\frac{C}{\left|\omega_{n}\right|^{4}}=\tilde{C}_{n}
$$

But

$$
a_{n} \geq \sum_{k=0}^{n-1}\left(a_{k+1}-b_{k}\right) \geq m n .
$$

Hence if $\left|\omega_{n}\right| \geq c \mathrm{e}^{-c^{\prime} n}$, then (4.1) is satisfied.

\subsection{Example 3}

Now we are interested in the case where the lengths of the uncontrolled parts $\left(b_{n}, a_{n+1}\right)_{n}$ go to infinity, and the length of the controlled parts go to zero. Since $a_{n+1}-b_{n} \geq m n^{\alpha}$, we have that

$$
a_{n} \geq \sum_{k=0}^{n-1}\left(a_{k+1}-b_{k}\right) \geq \sum_{k=0}^{n-1} m n^{\alpha} \geq m^{\prime} n^{\alpha+1} .
$$

On the other hand, since $a_{n+1}-b_{n} \leq M n^{\beta}$, and $\left|\omega_{n}\right| \geq c \mathrm{e}^{-c^{\prime} n^{1+\alpha}}$, we see that (4.1) is satisfied if $2 \beta \leq \alpha+1$ (which implies $0 \leq \alpha \leq \beta \leq 1$, since $0 \leq \alpha \leq \beta$ ).

Similar results hold for $\rho_{1}$.

\section{TOOLS FOR THE $N$ Dimensional CASE}

The main thing that has to be proved is Proposition 2.2. Then Theorem 2.2 follows as for the one dimensional case. Proposition 2.2 follows from the following generalization of Lemma 3.1:

Lemma 5.1. For all $n$, there exists $C_{n}$ and $\tilde{C}_{n+1}$ such that the solution $w(t, x)$ of $(2.7)$ satisfies

$$
\int_{T / 4}^{3 T / 4} \int_{\mathcal{O}_{0}} w(t, x)^{2} \mathrm{~d} x \mathrm{~d} t \leq \tilde{C}_{1} \int_{0}^{T} \int_{\omega_{1}} w(t, x)^{2} \mathrm{~d} x \mathrm{~d} t
$$

and

$$
\int_{T / 4}^{3 T / 4} \int_{\mathcal{O}_{n}} w(t, x)^{2} \mathrm{~d} x \mathrm{~d} t \leq C_{n} \int_{0}^{T} \int_{\omega_{n}} w(t, x)^{2} \mathrm{~d} x \mathrm{~d} t+\tilde{C}_{n+1} \int_{0}^{T} \int_{\omega_{n+1}} w(t, x)^{2} \mathrm{~d} x \mathrm{~d} t .
$$

Moreover the constants $C_{n}$ and $\tilde{C}_{n+1}$ have the same value than in the one dimensional case, given by (3.3), and (3.4).

Lemma 5.1 follows from the generalization of Lemmas 3.2 and 3.3. We are going to prove the generalization of Lemma 3.2. The one of Lemma 3.3 is easy and left to the reader.

Now fix $n$, define $c_{n}, d_{n}, c_{n+1}^{\prime}$ and $d_{n+1}^{\prime}$ as in the one dimensional case, and construct a smooth (radial) cut-off function $\psi: \Omega \rightarrow \mathbb{R}_{+}$such that $0 \leq \psi \leq 1$ on $\Omega, \psi=1$ if $|x| \in\left(d_{n}, d_{n+1}^{\prime}\right)$ and $\psi=0$ if $|x| \notin\left(c_{n}, c_{n+1}^{\prime}\right)$. (Of course $\psi$ depends on $n$.) 
Let us now consider $z:=\psi(x) w$. The function $z(t, x)$ satisfies the following heat equation in the bounded domain $\Omega_{n}:=\left\{x,|x| \in\left(c_{n}, c_{n+1}^{\prime}\right)\right\}$ :

$$
\begin{cases}z_{t}+\Delta z=h(t, x), & x \in \Omega_{n}, t \in(0, T) \\ z(t, x)=0, & x \in \partial \Omega_{n}, t \in(0, T)\end{cases}
$$

where $h(t, x):=\operatorname{div}(w \nabla \psi)+\nabla \psi \cdot \nabla w \in L^{2}\left(\Omega_{n} \times(0, T)\right)$. Note also that the function $h$ is supported in space in $\left\{|x| \in\left(c_{n}, d_{n}\right) \cup\left(d_{n+1}^{\prime}, c_{n+1}^{\prime}\right)\right\}$. We denote $\lambda_{n}:=c_{n+1}^{\prime}-c_{n}$ the length of the interval $\left(c_{n}, c_{n+1}\right)$, and

$$
\theta(t):=\frac{1}{t(T-t)}, \quad \phi(x):=|x|-c_{n}, \quad \sigma(t, x):=\theta(t)\left(\mathrm{e}^{2 S\|\phi\|_{\infty}}-\mathrm{e}^{S \phi(x)}\right)
$$

( $S$ will be chosen equal to $\left.1 / \lambda_{n}\right)$. We prove the following

Lemma 5.2. The solution $z$ of (5.3) satisfies the observability estimate:

$$
\int_{T / 4}^{3 T / 4} \int_{\Omega_{n}} z(t, x)^{2} \mathrm{~d} x \mathrm{~d} t \leq K\left(\lambda_{n}, T\right) \int_{0}^{T} \int_{\Omega_{n}} \mathrm{e}^{-2 R\left(\lambda_{n}, T\right) \sigma(t, x)} h(t, x)^{2} \mathrm{~d} x \mathrm{~d} t
$$

where the constants $K\left(\lambda_{n}, T\right)$ and $R\left(\lambda_{n}, T\right)$ have the same value than in the one dimensional case (3.8) and (3.9). Proof of Lemma 5.2. Consider

$$
v(t, x):=\mathrm{e}^{-R \sigma(t, x)} z(t, x)
$$

Denote $\Gamma_{n}:=\partial \Omega_{n}$. The function $v$ satisfies

$$
\begin{cases}P_{R} v:=\mathrm{e}^{-R \sigma(t, x)}\left(\partial_{t}+\Delta\right)\left(\mathrm{e}^{R \sigma} v\right)=\mathrm{e}^{-R \sigma} f & x \in \Omega_{n}, t \in(0, T), \\ v=0 & x \in \Gamma_{n}, t \in(0, T) \\ \partial_{\nu} v=\mathrm{e}^{-R \sigma(t, x)} \partial_{\nu} z=0 & x \in \Gamma_{n}, t \in(0, T) \\ v(t=0)=0=v(t=T) & x \in \Omega_{n}\end{cases}
$$

We are going to prove

$$
\int_{0}^{T} \int_{\Omega_{n}} v(t, x)^{2} \mathrm{~d} x \mathrm{~d} t \leq C(R, S)\left\|\mathrm{e}^{-R \sigma} h\right\|_{0}^{2}
$$

for some suitable $C(R, S)$, which will imply (5.4). Let us decompose the operator $P_{R} v$ in two parts:

$$
P_{R} v=P_{R}^{+} v+P_{R}^{-} v
$$

with

$$
\begin{gathered}
P_{R}^{+} v:=R\left(\sigma_{t}+R|\nabla \sigma|^{2}\right) v+\Delta v, \\
P_{R}^{-} v:=v_{t}+R(\nabla \sigma \cdot \nabla v+\operatorname{div}(v \nabla \sigma)) .
\end{gathered}
$$

Then

where

$$
\left\|\mathrm{e}^{-R \sigma} h\right\|_{0}^{2}=\left\|P_{R} v\right\|_{0}^{2}=\left\|P_{R}^{+} v\right\|_{0}^{2}+\left\|P_{R}^{-} v\right\|_{0}^{2}+2\left\langle P_{R}^{+} v, P_{R}^{-} v\right\rangle
$$

$$
\|v\|_{0}^{2}:=\int_{0}^{T} \int_{\Omega_{n}} v(t, x)^{2} \mathrm{~d} x \mathrm{~d} t=\iint_{Q_{T}} v(t, x)^{2} \mathrm{~d} x \mathrm{~d} t .
$$

The proof of (5.7) is based on a the estimate (by below) of the scalar product $\left\langle P_{R}^{+} v, P_{R}^{-} v\right\rangle$. 


\subsection{Computation of the scalar product}

We denote

$$
\rho:=R S \theta \mathrm{e}^{S \phi}
$$

Let us compute the scalar product between $P_{R}^{+}$and $P_{R}^{-}$:

Lemma 5.3. We have the following identity:

$$
\begin{aligned}
\left\langle P_{R}^{+}, P_{R}^{-}\right\rangle= & P_{1}+P_{2}+P_{3}+P_{4} \\
= & \iint_{Q_{T}} 2 \rho\left(D^{2} \phi \cdot \nabla v\right) \cdot \nabla v+2 S \rho|\nabla \phi \cdot \nabla v|^{2} \\
& +\iint_{Q_{T}} v^{2}\left(2 S \rho^{3}|\nabla \phi|^{4}+\rho^{3} \nabla \phi \cdot \nabla\left(|\nabla \phi|^{2}\right)+\frac{R}{2} \Delta^{2} \sigma-\frac{R}{2} \sigma_{t t}-2 R^{2} \nabla \sigma \cdot \nabla \sigma_{t}\right) .
\end{aligned}
$$

Proof of Lemma 5.3.

$$
\left\langle P_{R}^{+}, P_{R}^{-}\right\rangle=\left\langle R\left(\sigma_{t}+R|\nabla \sigma|^{2}\right) v+\Delta v, v_{t}+R(\nabla \sigma \cdot \nabla v+\operatorname{div}(v \nabla \sigma))\right\rangle=P_{1}+P_{2}+P_{3}+P_{4},
$$

with

$$
\begin{aligned}
& P_{1}:=\left\langle R\left(\sigma_{t}+R|\nabla \sigma|^{2}\right) v+\Delta v, v_{t}\right\rangle, \\
& P_{2}:=R^{2}\left\langle\sigma_{t} v, \nabla \sigma \cdot \nabla v+\operatorname{div}(v \nabla \sigma)\right\rangle, \\
& P_{3}:=R^{3}\left\langle|\nabla \sigma|^{2} v, \nabla \sigma \cdot \nabla v+\operatorname{div}(v \nabla \sigma)\right\rangle \\
& P_{4}:=R\langle\Delta v, \nabla \sigma \cdot \nabla v+\operatorname{div}(v \nabla \sigma)\rangle .
\end{aligned}
$$

Let us study each term:

First term: $P_{1}$

$$
\begin{aligned}
P_{1} & =\iint_{Q_{T}} R\left(\sigma_{t}+R|\nabla \sigma|^{2}\right) v v_{t}+v_{t} \Delta v \\
& =\iint_{Q_{T}} R\left(\sigma_{t}+R|\nabla \sigma|^{2}\right)\left(\frac{1}{2} v^{2}\right)_{t}-\nabla v_{t} \cdot \nabla v \\
& =\left[\int_{\Omega} \frac{R}{2}\left(\sigma_{t}+R|\nabla \sigma|^{2}\right) v^{2}\right]_{0}^{T}-\iint_{Q_{T}} \frac{R}{2}\left(\sigma_{t}+R|\nabla \sigma|^{2}\right)_{t} v^{2}+\frac{1}{2}\left(|\nabla v|^{2}\right)_{t} \\
& =\left[\int_{\Omega} \frac{R}{2}\left(\sigma_{t}+R|\nabla \sigma|^{2}\right) v^{2}-\frac{1}{2}|\nabla v|^{2}\right]_{0}^{T}-\iint_{Q_{T}} \frac{R}{2}\left(\sigma_{t}+R|\nabla \sigma|^{2}\right)_{t} v^{2} .
\end{aligned}
$$

Thanks to the term $\mathrm{e}^{-R \sigma}$, we obtain that

$$
P_{1}=-\iint_{Q_{T}} \frac{R}{2}\left(\sigma_{t}+R|\nabla \sigma|^{2}\right)_{t} v^{2} .
$$


Second term: $P_{2}$

$$
\begin{aligned}
P_{2}: & =R^{2} \iint_{Q_{T}} \sigma_{t} v(\nabla \sigma \cdot \nabla v+\operatorname{div}(v \nabla \sigma)) \\
& =R^{2} \iint_{Q_{T}} \sigma_{t} v(2 \nabla \sigma \cdot \nabla v+v \Delta \sigma) \\
& =R^{2} \iint_{Q_{T}} \sigma_{t} \nabla \sigma \cdot \nabla\left(v^{2}\right)+\sigma_{t}(\Delta \sigma) v^{2} \\
& =-R^{2} \iint_{Q_{T}} \operatorname{div}\left(\sigma_{t} \nabla \sigma\right) v^{2}+R^{2} \iint_{Q_{T}} \sigma_{t}(\Delta \sigma) v^{2}
\end{aligned}
$$

Hence

$$
P_{2}=-R^{2} \iint_{Q_{T}} v^{2} \nabla \sigma \cdot \nabla \sigma_{t}
$$

Third term: $P_{3}$

$$
\begin{aligned}
P_{3}: & =R^{3} \iint_{Q_{T}}|\nabla \sigma|^{2} v(\nabla \sigma \cdot \nabla v+\operatorname{div}(v \nabla \sigma)) \\
& =R^{3} \iint_{Q_{T}} 2|\nabla \sigma|^{2} \nabla \sigma \cdot \nabla\left(\frac{1}{2} v^{2}\right)+|\nabla \sigma|^{2}(\Delta \sigma) v^{2} \\
& =R^{3} \iint_{Q_{T}}-\operatorname{div}\left(|\nabla \sigma|^{2} \nabla \sigma\right) v^{2}+|\nabla \sigma|^{2}(\Delta \sigma) v^{2} \\
& =R^{3} \iint_{Q_{T}}-v^{2} \nabla\left(|\nabla \sigma|^{2}\right) \cdot \nabla \sigma
\end{aligned}
$$

Let us compute this last term:

$$
\begin{aligned}
-R^{3} \nabla\left(|\nabla \sigma|^{2}\right) \cdot \nabla \sigma & =-R^{3} \nabla\left(S^{2} \theta^{2} \mathrm{e}^{2 S \phi}|\nabla \phi|^{2}\right) \cdot\left(-S \theta \mathrm{e}^{S \phi} \nabla \phi\right) \\
& =R^{3} S^{3} \theta^{3} \mathrm{e}^{S \phi} \nabla\left(\mathrm{e}^{2 S \phi}|\nabla \phi|^{2}\right) \cdot \nabla \phi \\
& =R^{3} S^{3} \theta^{3} \mathrm{e}^{S \phi}\left(2 S \mathrm{e}^{2 S \phi}|\nabla \phi|^{2} \nabla \phi+\mathrm{e}^{2 S \phi} \nabla\left(|\nabla \phi|^{2}\right)\right) \cdot \nabla \phi \\
& =R^{3} S^{3} \theta^{3} \mathrm{e}^{3 \phi}\left(2 S|\nabla \phi|^{4}+\nabla\left(|\nabla \phi|^{2}\right) \cdot \nabla \phi\right) \\
& =\rho^{3}\left(2 S|\nabla \phi|^{4}+\nabla\left(|\nabla \phi|^{2}\right) \cdot \nabla \phi\right) .
\end{aligned}
$$

Hence

$$
P_{3}=\iint_{Q_{T}} 2 S \rho^{3}|\nabla \phi|^{4} v^{2}+\rho^{3} \nabla \phi \cdot \nabla\left(|\nabla \phi|^{2}\right) v^{2} .
$$

$\underline{\text { Fourth term: } P_{4}}$

$$
\begin{aligned}
P_{4} & =R \iint_{Q_{T}} \Delta v(\nabla \sigma \cdot \nabla v+\operatorname{div}(v \nabla \sigma)) \\
& =R \int_{0}^{T} \int_{\Gamma_{n}} \partial_{\nu} v(\nabla \sigma \cdot \nabla v+\operatorname{div}(v \nabla \sigma))-R \iint_{Q_{T}} \nabla v \cdot \nabla(\nabla \sigma \cdot \nabla v+\operatorname{div}(v \nabla \sigma)) \\
& =-R \iint_{Q_{T}} \nabla v \cdot \nabla(\nabla \sigma \cdot \nabla v+\operatorname{div}(v \nabla \sigma)) .
\end{aligned}
$$


With the repeated indexes convention, we have

$$
\begin{aligned}
-R \iint_{Q_{T}} \nabla v \cdot \nabla(\nabla \sigma \cdot \nabla v+\operatorname{div}(v \nabla \sigma)) & =-R \iint_{Q_{T}} \partial_{i} v \partial_{i}\left(2 \partial_{j} \sigma \partial_{j} v+v \Delta \sigma\right) \\
& =-R \iint_{Q_{T}} 2 \partial_{i} \partial_{j} \sigma \partial_{i} v \partial_{j} v+2 \partial_{j} \sigma \partial_{i} v \partial_{i} \partial_{j} v+(\Delta \sigma)|\nabla v|^{2}+v \partial_{i} v \partial_{i} \Delta \sigma
\end{aligned}
$$

For the first term, we obtain that

$$
\begin{aligned}
-2 R \partial_{i} \partial_{j} \sigma \partial_{i} v \partial_{j} v=2 R S \partial_{i} \partial_{j} \phi \theta \mathrm{e}^{S \phi} \partial_{i} v \partial_{j} v+2 R S^{2} \partial_{i} \phi \partial_{j} \phi \theta \mathrm{e}^{S \phi} \partial_{i} v \partial_{j} v & =2 \rho\left(D^{2} \phi \cdot \nabla v\right) \cdot \nabla v+2 S \rho|\nabla \phi \cdot \nabla v|^{2} \\
& =2 .
\end{aligned}
$$

Hence

$$
\begin{aligned}
P_{4} & =2 \iint_{Q_{T}} \rho\left(D^{2} \phi \cdot \nabla v\right) \cdot \nabla v+2 S \iint_{Q_{T}} \rho|\nabla \phi \cdot \nabla v|^{2}-R \iint_{Q_{T}} \nabla \sigma \cdot \nabla\left(|\nabla v|^{2}\right)+(\Delta \sigma)|\nabla v|^{2}+\nabla(\Delta \sigma) \cdot \nabla\left(\frac{1}{2} v^{2}\right) \\
& =2 \iint_{Q_{T}} \rho\left(D^{2} \phi \cdot \nabla v\right) \cdot \nabla v+2 S \iint_{Q_{T}} \rho|\nabla \phi \cdot \nabla v|^{2}+R \iint_{Q_{T}} \frac{1}{2}\left(\Delta^{2} \sigma\right) v^{2}
\end{aligned}
$$

And this implies that

$$
\begin{aligned}
\left\langle P_{R}^{+}, P_{R}^{-}\right\rangle= & P_{1}+P_{2}+P_{3}+P_{4} \\
= & \iint_{Q_{T}} 2 \rho\left(D^{2} \phi \cdot \nabla v\right) \cdot \nabla v+2 S \rho|\nabla \phi \cdot \nabla v|^{2} \\
& +\iint_{Q_{T}} v^{2}\left(2 S \rho^{3}|\nabla \phi|^{4}+\rho^{3} \nabla \phi \cdot \nabla\left(|\nabla \phi|^{2}\right)+\frac{R}{2} \Delta^{2} \sigma-\frac{R}{2} \sigma_{t t}-2 R^{2} \nabla \sigma \cdot \nabla \sigma_{t}\right) .
\end{aligned}
$$

\subsection{Some simplifications due to the choice of the function $\phi$}

We have chosen

$$
\phi(x):=|x|-c_{n} .
$$

Then denoting $r:=|x|$, we have that

$$
\nabla \phi(x)=\frac{x}{r}, \quad|\nabla \phi(x)|=1,
$$

hence

Moreover

$$
2 S \rho^{3}|\nabla \phi|^{4}+\rho^{3} \nabla \phi \cdot \nabla\left(|\nabla \phi|^{2}\right)=2 S \rho^{3} .
$$

hence

$$
\partial_{i} \partial_{j} \phi(x)=\frac{1}{r} \delta_{i j}-\frac{1}{r^{3}} x_{i} x_{j},
$$

We deduce that

$$
\Delta \phi(x)=0, \quad\left(D^{2} \phi(x) \cdot \xi\right) \cdot \xi=\frac{1}{r}|\xi|^{2}-\frac{1}{r^{3}}(x \cdot \xi)^{2} \geq 0 .
$$

$$
-2 R^{2} \nabla \sigma \cdot \nabla \sigma_{t}=-2 R^{2}\left(-\theta S \mathrm{e}^{S \phi} \nabla \phi\right) \cdot\left(-\theta_{t} S \mathrm{e}^{S \phi} \nabla \phi\right)=-2 R^{2} \theta \theta_{t} S^{2} \mathrm{e}^{2 S \phi},
$$

and

$$
\Delta \sigma(t, x)=-\theta S^{2} \mathrm{e}^{S \phi}, \quad \frac{R}{2} \Delta^{2} \sigma(t, x)=-R \theta S^{4} \mathrm{e}^{S \phi}
$$


Then we deduce from the identity (5.11) that

$$
\left\langle P_{R}^{+}, P_{R}^{-}\right\rangle \geq \iint_{Q_{T}} v^{2}\left(2 S \rho^{3}-R \theta S^{4} \mathrm{e}^{S \phi}-\frac{R}{2} \theta_{t t}\left(\mathrm{e}^{2 S\|\phi\|_{\infty}}-\mathrm{e}^{S \phi(x)}\right)-2 R^{2} \theta \theta_{t} S^{2} \mathrm{e}^{2 S \phi}\right) .
$$

\subsection{Choice of the parameters $\boldsymbol{R}$ and $\boldsymbol{S}$}

We need to bound from below the coefficient of $v^{2}$ in (5.22). This will be done with a good choice of the parameters $R$ and $S$.

Lemma 5.4. Consider

$$
S=\frac{1}{\max \left(1, c_{n+1}^{\prime}-c_{n}\right)} \quad \text { and } \quad R=\frac{R_{0}}{S^{2}}=R_{0} \max \left(1, c_{n+1}^{\prime}-c_{n}\right)^{2}
$$

for some $R_{0}$. Then we can choose $R_{0}$ (independent of $n$ ) such that

$$
R \theta S^{4} \mathrm{e}^{S \phi}+\frac{R}{2} \theta_{t t}\left(\mathrm{e}^{2 S\|\phi\|_{\infty}}-\mathrm{e}^{S \phi(x)}\right)+2 R^{2} \theta \theta_{t} S^{2} \mathrm{e}^{2 S \phi} \leq S \rho^{3} .
$$

Note that (5.23) and (5.22) imply that

$$
\left\langle P_{R}^{+}, P_{R}^{-}\right\rangle \geq \iint_{Q_{T}} S \rho^{3} v^{2}
$$

Proof of Lemma 5.4. We want to prove that

$$
\frac{1}{S \rho^{3}}\left(R \theta S^{4} \mathrm{e}^{S \phi}+\frac{R}{2} \theta_{t t}\left(\mathrm{e}^{2 S\|\phi\|_{\infty}}-\mathrm{e}^{S \phi(x)}\right)+2 R^{2} \theta \theta_{t} S^{2} \mathrm{e}^{2 S \phi}\right) \leq 1
$$

or equivalently

$$
\frac{1}{R^{2} \theta^{2}} \mathrm{e}^{-2 S \phi(x)}+\frac{1}{2 R^{2} S^{4}} \frac{\theta_{t t}}{\theta^{3}}\left(\mathrm{e}^{2 S\|\phi\|_{\infty}}-\mathrm{e}^{S \phi(x)}\right) \mathrm{e}^{-3 S \phi(x)}+2 \frac{1}{R S^{2}} \frac{\theta_{t}}{\theta^{2}} \mathrm{e}^{-S \phi(x)} \leq 1 .
$$

This is clear: indeed, $x \mapsto S \phi(x)$ varies between 0 and 1 , moreover $\theta_{t} \leq c \theta^{2}$ and $\theta_{t t} \leq c \theta^{3}$ for some constant $c$, and at last $R S^{2}=R_{0}$. Then it is sufficient to choose $R_{0}$ large enough.

\subsection{End of the proof}

We deduce from the previous computations that

$$
\left\|\mathrm{e}^{-R \sigma} h\right\|_{0}^{2} \geq 2\left\langle P_{R}^{+}, P_{R}^{-}\right\rangle \geq 2 \iint_{Q_{T}} S \rho^{3} v^{2}=2 \iint_{Q_{T}} R_{0}^{2} R \theta^{3} \mathrm{e}^{3 S \phi} z^{2} \mathrm{e}^{-2 R \sigma} \geq 2 c_{1} R \mathrm{e}^{-c_{2} R} \int_{T / 4}^{T / 4} \int_{\Omega_{n}} z^{2}
$$

which gives (5.4).

\section{Appendix}

To prove that $\tilde{A}$ is the generator of an analytic semi-group in $L_{\rho_{2}}^{2}(\Omega)$, the main step consists into proving that the following problem

$$
\left\{\begin{array}{l}
\lambda u-u_{x x}=f \in L_{\rho_{2}}^{2}(\Omega) \\
u(x=0)=0
\end{array}\right.
$$


has the following property: there exists $\lambda_{0} \geq 0$ such that for all $\lambda \in \mathbb{C}, \operatorname{Re} \lambda>\lambda_{0}$, then the problem (6.1) has one and only one solution $u \in D(\tilde{A})$, and $u$ satisfies

$$
\|u\|_{\rho_{2}} \leq \frac{C}{|\lambda|}\|f\|_{\rho_{2}}
$$

First let us suppose that (6.1) has one and only one solution, and we prove that it has to verify (6.2): multiplying (6.1) by $\rho_{2} \bar{u}$ and integrating on $\Omega$ leads to

$$
\lambda \int_{\Omega}|u|^{2} \rho_{2}+\int_{\Omega}\left(\rho_{2} \bar{u}\right)_{x} u_{x}=\int_{\Omega} \rho_{2} \bar{u} f .
$$

Hence

$$
\lambda \int_{\Omega}|u|^{2} \rho_{2}+\int_{\Omega} \rho_{2}\left|u_{x}\right|^{2}=\int_{\Omega} \rho_{2} \bar{u} f-\int_{\Omega} \rho_{2, x} \bar{u} u_{x} .
$$

Taking the real part and using the differential inequality (2.5) satisfied by $\rho_{2}$, we obtain

$$
\operatorname{Re} \lambda \int_{\Omega}|u|^{2} \rho_{2}+\int_{\Omega} \rho_{2}\left|u_{x}\right|^{2} \leq \frac{1}{2} \int_{\Omega} \rho_{2}|f|^{2}+\frac{1}{2} \int_{\Omega} \rho_{2}|u|^{2}+\frac{1}{2} \int_{\Omega} \rho_{2}\left|u_{x}\right|^{2}+\frac{\alpha_{2}^{2}}{2} \int_{\Omega} \rho_{2}|u|^{2} .
$$

Hence

$$
\left(\operatorname{Re} \lambda-\frac{1}{2}-\frac{\alpha_{2}^{2}}{2}\right) \int_{\Omega}|u|^{2} \rho_{2}+\int_{\Omega} \rho_{2}\left|u_{x}\right|^{2} \leq \frac{1}{2} \int_{\Omega} \rho_{2}|f|^{2}
$$

thus for all $\lambda$ such that $\operatorname{Re} \lambda>\left(1+\alpha_{2}^{2}\right) / 2$, we have the a priori estimate

$$
\int_{\Omega} \rho_{2}\left|u_{x}\right|^{2} \leq \frac{1}{2} \int_{\Omega} \rho_{2}|f|^{2}
$$

Now we multiply (6.3) by $\bar{\lambda}$, and we obtain

$$
|\lambda|^{2} \int_{\Omega}|u|^{2} \rho_{2}+\bar{\lambda} \int_{\Omega} \rho_{2}\left|u_{x}\right|^{2}=\bar{\lambda} \int_{\Omega} \rho_{2} \bar{u} f-\bar{\lambda} \int_{\Omega} \rho_{2, x} u_{x} \bar{u}
$$

Since $\operatorname{Re} \lambda \geq 0$ we have

$$
\begin{aligned}
|\lambda|^{2} \int_{\Omega}|u|^{2} \rho_{2} \leq\left|\bar{\lambda} \int_{\Omega} \rho_{2} \bar{u} f-\bar{\lambda} \int_{\Omega} \rho_{2, x} u_{x} \bar{u}\right| & \\
& \leq\left(\int_{\Omega} \rho_{2}|f|^{2}\right)^{1 / 2}\left(|\lambda|^{2} \int_{\Omega} \rho_{2}|u|^{2}\right)^{1 / 2}+\alpha_{2}\left(\int_{\Omega} \rho_{2}\left|u_{x}\right|^{2}\right)^{1 / 2}\left(|\lambda|^{2} \int_{\Omega} \rho_{2}|u|^{2}\right)^{1 / 2} .
\end{aligned}
$$

Hence using (6.4), we obtain that

$$
|\lambda|\|u\|_{\rho_{2}} \leq\|f\|_{\rho_{2}}+\alpha_{2}\left\|u_{x}\right\|_{\rho_{2}} \leq\left(1+\frac{\alpha_{2}}{\sqrt{2}}\right)\|f\|_{\rho_{2}},
$$

which concludes the proof of (6.2).

Now it remains to prove that the problem (6.1) has one and only one solution $u \in D(\tilde{A})$. The uniqueness of the solution follows directly from (6.2). We prove the existence of a solution by approximation: given $f \in L_{\rho_{2}}^{2}(\Omega)$, 
consider the sequence of functions $\left(f_{n}\right)_{n}$ defined by: $f_{n}(x)=f(x)$ if $x \leq n$, and $f_{n}(x)=0$ otherwise. Every function $f_{n}$ is compactly supported, and belongs to $L^{2}(\Omega)$. Consider $u_{n}$ the only solution of the problem

$$
\left\{\begin{array}{l}
\lambda u-u_{x x}=f_{n} \in L^{2}(\Omega), \\
u(x=0)=0 .
\end{array}\right.
$$

Then $u_{n} \in H^{2}(\Omega)$ and satisfies the estimate

$$
|\lambda|\left\|u_{n}\right\|_{\rho_{2}}+\left\|u_{n, x}\right\|_{\rho_{2}}+\left\|u_{n, x x}\right\|_{\rho_{2}} \leq C\|f\|_{\rho_{2}}
$$

if Re $\lambda$ is large enough. We deduce that the sequence $\left(u_{n}\right)_{n}$ satisfies the Cauchy criterion in $H_{\rho_{2}}^{2}(\Omega)$, which is of course a complete space, therefore the sequence $\left(u_{n}\right)_{n}$ converges to some $u \in H_{\rho_{2}}^{2}(\Omega)$, which is solution of (6.1).

Acknowledgements. The authors are grateful to the referee and to Enrique Zuazua for helping to improve the quality of this paper with valuable comments and suggestions.

\section{REFERENCES}

[1] P. Albano and P. Cannarsa, Lectures on Carleman estimates for elliptic and parabolic operators with applications (in preparation).

[2] S. Aniţa and V. Barbu, Null controllability of nonlinear convective heat equations. ESAIM: COCV 5 (2000) 157-173.

[3] V.R. Cabanillas, S.B. De Menezes and E. Zuazua, Null controllability in unbounded domains for the semilinear heat equation with nonlinearities involving gradient terms. J. Optim. Theory Appl. 110 (2001) 245-264.

[4] P. Cannarsa, P. Martinez and J. Vancostenoble, Nulle contrôlabilité régionale pour des équations de la chaleur dégénérées. Comptes Rendus Mécanique 330 (2002) 397-401.

[5] L. De Teresa, Approximate controllability of a semilinear heat equation in $\mathbb{R}^{n}$. SIAM J. Control Optim. 36 (1998) $2128-2147$.

[6] L. De Teresa and E. Zuazua, Approximate controllability of the semilinear heat equation in unbounded domains. Nonlinear Anal. TMA 37 (1999) 1059-1090.

[7] Sz. Dolecki and D.L. Russell, A general theory of observation and control. SIAM J. Control Optim. 15 (1977) 185-220.

[8] C. Fabre, J.P. Puel and E. Zuazua, Approximate controllability of the semilinear heat equation. Proc. Roy. Soc. Edinb. A 125 (1995) $185-220$.

[9] H.O. Fattorini and D.L. Russell, Exact controllability theorems for linear parabolic equations in one space dimension. Arch. Rat. Mech. Anal. 4 (1971) 272-292.

[10] H.O. Fattorini and D.L. Russell, Uniform bounds on biorthogonal functions for real exponentials with an application to the control theory of parabolic equations. Quart. Appl. Math. 32 (1974) 45-69.

[11] E. Fernández-Cara, Null controllability of the semilinear heat equation. ESAIM: COCV 2 (1997) 87-103.

[12] E. Fernández-Cara and E. Zuazua, The cost of approximate controllability for heat equations: the linear case. Adv. Differ. Equations 5 (2000) 465-514.

[13] E. Fernández-Cara and E. Zuazua, Controllability for weakly blowing-up semilinear heat equations. Ann. Inst. H. Poincaré Anal. Non Linéaire 17 (2000) 583-616.

[14] A.V. Fursikov and O. Yu Imanuvilov, Controllability of evolution equations, Seoul National University, Seoul, Korea. Lect. Notes Ser. 34 (1996).

[15] O. Yu. Imanuvilov, Boundary controllability of parabolic equations. Russian Acad. Sci. Sb. Math. 186 (1995) $109-132$.

[16] B.F. Jones Jr., A fundamental solution for the heat equation which is supported in a strip. J. Math. Anal. Appl. 60 (1977) 314-324.

[17] A. Khapalov, Mobile points controls versus locally distributed ones for the controllability of the semilinear parabolic equations. SIAM J. Control Optim. 40 (2001) 231-252.

[18] I. Lasiecka and R. Triggiani, Carleman estimates and exact boundary controllability for a system of coupled, non conservative second order hyperbolic equations, in Partial Differential Equations Methods in Control and Shape Analysis. Marcel Dekker, New York, Lect. Notes Pure Appl. Math. 188 (1994) 215-243.

[19] G. Lebeau and L. Robbiano, Contrôle exact de l'équation de la chaleur. Comm. Partial Differ. Equations 20 (1995) 335-356.

[20] S. Micu and E. Zuazua, On the lack of null controllability of the heat equation on the half-line. Trans. Amer. Math. Soc. 353 (2001) 1635-1659.

[21] S. Micu and E. Zuazua, On the lack of null controllability of the heat equation on the half-space. Portugaliae Math. 58 (2001) 1-24. 
[22] L. Rosier, Exact boundary controllability for the linear Korteweg-de Vries equation on the half-line. SIAM J. Control Optim. 39 (2000) 331-351.

[23] D.L. Russell, A unified boundary controllability theory for hyperbolic and parabolic partial differential equations. Stud. Appl. Math. 52 (1973) 189-221.

[24] D. Tataru, A priori estimates of Carleman's type in domains with boundary. J. Math. Pures Appl. 73 (1994) 355-387.

[25] D. Tataru, Carleman estimates and unique continuation near the boundary for P.D.E.'s. J. Math. Pures Appl. 75 367-408 ((1996).

[26] X. Zhang, A remark on null controllability of the heat equation. SIAM J. Control Optim. 40 (2001) 39-53.

[27] E. Zuazua, Approximate controllability for the semilinear heat equation with globally Lipschitz nonlinearities. Control Cybern. 28 (1999) 665-683. 